\title{
OPEN Soil moisture remote sensing using SIW cavity based metamaterial perfect absorber
}

\author{
Majid Amiri ${ }^{1,2}$, Mehran Abolhasan ${ }^{1}$, Negin Shariati ${ }^{1,2}$ \& Justin Lipman ${ }^{1,2}$
}

Continuous and accurate sensing of water content in soil is an essential and useful measure in the agriculture industry. Traditional sensors developed to perform this task suffer from limited lifetime and also need to be calibrated regularly. Further, maintenance, support, and deployment of these sensors in remote environments provide additional challenges to the use of conventional soil moisture sensors. In this paper, a metamaterial perfect absorber (MPA) based soil moisture sensor is introduced. The ability of MPAs to absorb electromagnetic signals with near $100 \%$ efficiency facilitates the design of highly accurate and low-profile radio frequency passive sensors. MPA based sensor can be fabricated from highly durable materials and can therefore be made more resilient than traditional sensors. High resolution sensing is achieved through the creation of physical channels in the substrate integrated waveguide (SIW) cavity. The proposed sensor does not require connection for both electromagnetic signals or for adding a testing sample. Importantly, an external power supply is not needed, making the MPA based sensor the perfect solution for remote and passive sensing in modern agriculture. The proposed MPA based sensor has three absorption bands due to the various resonance modes of the SIW cavity. By changing the soil moisture level, the absorption peak shifts by $10 \mathrm{MHz}$, $23.3 \mathrm{MHz}$, and $60 \mathrm{MHz}$, which is correlated with the water content percentage at the first, second and third absorption bands, respectively. Finally, a $6 \times 6$ cell array with a total size of $312 \mathrm{~mm} \times 312 \mathrm{~mm}$ has been fabricated and tested. A strong correlation between measurement and simulation results validates the design procedure.

Landy introduced metamaterial perfect absorbers (MPA) in $2008^{1}$. This specific classification of FSS metamaterial absorbs the incident waves with near-unity efficiency. The first reported MPA structure consisted of a dielectric substrate, resonator on top, and another metal layer at the substrate's backside. The resonator provides the electric response of MPA facing incident electromagnetic waves. Hence, the resonator's shape and structure specify the different electric behavior targeted in an application. The interaction of the resonator and metallic layer on two substrate sides provides the structure's magnetic response. Having described the MPA responses, different modifications could be applied to conventional MPA. For instance, a multi wideband absorption using multi resonators $\mathrm{s}^{2,3}$, and multi-layer structure ${ }^{4-6}$ have been reported in several articles. The initial purpose of conventional MPA was replacing heavy, expensive, and low efficiency traditional absorbers such as Ferrit and high loss sheets. Beyond the early implementations, MPA's capabilities with different absorption characteristics combined with a low profile and lightweight enable many new applications. Undesired signals absorbers ${ }^{7-10}$, thermal emitters ${ }^{11,12}$, terahertz applications ${ }^{13-15}$, optical switches ${ }^{16,17}$, energy harvesters ${ }^{18,19}$ and sensors ${ }^{4,20}$ are some of the popular applications of MPAs. It is worth noting that high absorption efficiency is a common requirement in all mentioned applications.

Sensors are a crucial part of modern technology in a wide variety of applications such as medicine, imaging, and agriculture. MPAs are a promising candidate to implement highly sensitive sensors. This is due to their flexibility to work at a wide range of frequency spectrum. A large number of MPAs have been introduced as a sensor in terahertz frequencies ${ }^{21-24}$. However, several restrictions, such as the complicated fabrication process, and the high cost of stable material in the terahertz spectrum, make implementing these sensors challenging. Moreover, the integration of terahertz sensors with other electrical devices is a further challenge due to the differences in size and operating frequency.

Microwave MPA sensors can overcome the above challenges. Several methods have been investigated to design MPA sensors at the microwave frequency spectrum. Implementing microfluidics channels in the structure 
has been reported in several articles. The channels are filled by injecting liquid or gas. It changes the substrate properties based on effective medium theory ${ }^{25}$. Consequently, the changes in resonance frequency are taken as a sensing parameter. It should be noted that the MPA needs to be calibrated prior to the sensing process. The channels are created in the additional layer attached to the MPA structure. Polydimethylsiloxane (PDMS) is a common material to make an extra layer that includes the channels ${ }^{26,27}$. The structures, which take advantage of this method are mostly complicated and need one or two connections for sensing. These characteristics could limit reported MPA sensors in large scale applications.

Another reported technique to use MPA as a microwave sensor is adding an analyte layer to the MPA structure ${ }^{28-30}$. In this method, an air gap can be added between the substrate and metal layer ${ }^{31}$ or between two substrates ${ }^{32,33}$. Like the previous method, the combination of the dielectric substrate and the sample provides an effective medium with a different permittivity $\left(\varepsilon_{\text {eff }}\right)$. However, fabricating a multi-layer structure, including an air layer to place the sample in the metamaterial structure, is challenging.

In this paper, a cavity structure has been implemented to design a unit cell of MPA. The cavity has been widely used in various applications, such as antennas and filters, to improve their characteristics ${ }^{34-38}$. The capability of trapping electromagnetic signal and high Q-factor makes the cavity resonator a promising candidate for MPA structure in sensing applications. However, the fabrication complexity and 3D structure of cavity structure are challenges to implementing cavity MPA. Substrate integrated waveguide (SIW) technology can overcome the mentioned challenges of traditional cavities. Additional to the advantages of conventional cavities, the SIW structure offers a planar, compact, and low profile structure. Therefore, these properties lead to easing the integration of SIW cavity MPA with electrical circuits and passive components.

The high concentration of electric field energy in the SIW center cavity structure at its dominant resonance mode has been used in sensing applications. For instance, the proposed liquid material sensor in ${ }^{39}$ consists of two SIW cavities. The outer cavity reduces the size and increases the sensor's Q-factor, while the middle SIW cavity, along with a microfluidic channel located on top of it, improves the sensing resolution. However, in the mentioned reports, the sensors need to be connected to the spectrum analyzer in transmission line mode to investigate the frequency shift and sense the targeted parameters. This requirement limits the usage of the sensor to invasive and laboratory applications. In another approach, the SIW cavity has been combined into the antenna to use as a sensor ${ }^{40-42}$. In this approach, the under-tested material is placed in a created slot in the middle of the cavity. Using the antenna structure eliminates having two connections for sensing procedure. Also, it facilitates remote sensing applications. However, similar to the filter sensor structure, antenna sensors need an electrical connection to them.

Precision agriculture requires monitoring and controlling environmental parameters such as temperature, weather conditions, and soil nutrition. One of the most impactful parameters in farming is remote monitoring the soil moisture. Besides the direct effect of soil moisture on growing the plants, it can stabilize the added fertilizer into the farm ${ }^{43}$. In this paper, a low-profile MPA with one layer structure has been investigated to perform in sensing application at microwave spectrum. Using MPA is a promising solution for remote sensing with no need for an electrical contact to design a non-invasive sensor. In this approach, the reflected waves from the MPA surface are used as sensing parameters that do not directly connect to the spectrum analyzer. Hence, it can facilitate wireless sensing by keeping the signal analyzer far away from the sensors. SIW cavity resonator has been used as a unit cell of MPA to design highly sensitive soil moisture sensors. Instead of using an extra PDMS layer, channels have been created in the SIW cavity. With the advent of various resonance modes in cavity structure, high-resolution sensing has been achieved by implementing the proposed MPA-based sensor. Three first mode resonance frequencies have been considered as sensing parameters. Despite dropping the absorption efficiency in higher modes, the resolution of the sensor increased accordingly. Furthermore, the proposed structure shows decent insensitivity facing electromagnetic waves with different incident angles and is polarization angle insensitive.

\section{MPA principles and unit cell design}

The main principle to design an MPA is perfect impedance matching with air intrinsic impedance $Z_{0}=377$ $\Omega$. The perfect impedance matching guarantees the minimum wave reflection from structure surface $\left(\Gamma(\omega)=\left|S_{11}\right|^{2} \approx 0\right)$. However, minimum wave transmission is also required to have perfect absorption characteristics. Due to use of a metal film on the back side of the substrate the transmission coefficient of MPA is zero $\left(T(\omega)=\left|S_{21}\right|^{2}=0\right)$. Considering these two parameters, absorption efficiency is obtained as Eq. (1):

$$
A(\omega)=1-\Gamma(\omega)-T(\omega)=1-\left|S_{11}\right|^{2}-\left|S_{21}\right|^{2}=1-\left|S_{11}\right|^{2}
$$

In perfect impedance matching, the real and imaginary parts of $Z(\omega)$ have to be equal to 1 and 0 , respectively. In another approach, the absorption ratio can be calculated based on the difference between $Z_{\text {in }}$ of MPA and intrinsic air impedance, according to the Eq. (2).

$$
A(\omega)=1-\Gamma(\omega)=1-\left|\frac{Z_{\text {in }}(\omega)-Z_{0}}{Z_{\text {in }}(\omega)+Z_{0}}\right|^{2}
$$

where the input impedance of MPA $\left(Z_{\text {in }}(\omega)\right)$ is calculated based on effective permittivity and permeability of MPA. These two parameters are a function of electric and magnetic responses at MPA resonance frequency. Input impedance of MPA can be calculated based on S-parameters of structure ${ }^{44}$ utilizing Eq. (3): 


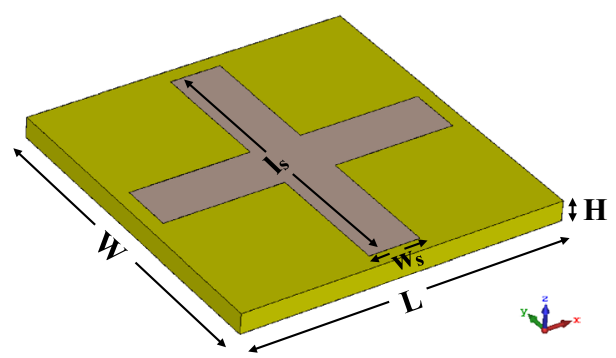

(a)

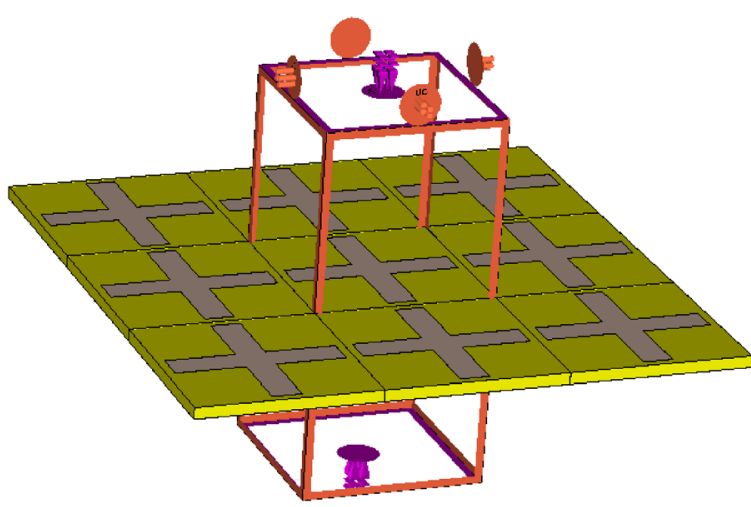

(b)

Figure 1. (a) Cavity resonator MPA unit cell , (b) Numerical setup to analyse MPA structure.

$$
Z_{\text {in }}(\omega)=\sqrt{\frac{\mu_{\text {eff }}(\omega)}{\varepsilon_{\text {eff }}(\omega)}}=\sqrt{\frac{\left(1+S_{11}(\omega)\right)^{2}-S_{21}^{2}(\omega)}{\left(1-S_{11}(\omega)\right)^{2}-S_{21}^{2}(\omega)}}
$$

Cavity resonator. A rectangular cavity is a short circuit ended rectangular waveguide. The reflection of the electromagnetic waves from conductive walls leads to creating a standing wave inside the cavity. The dimensions of the cavity resonator specify its characteristics. Different modes and mode numbers of electromagnetic waves $\left(T E_{m n p}\right.$ and $\left.T M_{m n p}\right)$ can be resonated in the rectangular cavity. The resonance frequency of the cavity is calculated according to the Eq. $(4)^{45}$ :

$$
f_{m n p}=\frac{C}{2 \sqrt{\varepsilon_{r} \mu_{r}}} \sqrt{\left(\frac{m}{L}\right)^{2}+\left(\frac{n}{H}\right)^{2}+\left(\frac{p}{W}\right)^{2}}
$$

where $C=3 \times 10^{11} \mathrm{~mm} / \mathrm{s}$ and $\varepsilon_{r}$ and $\mu_{r}$ are permittivity and permeability of dielectric inside the cavity. Moreover, $\mathrm{W}, \mathrm{L}$, and $\mathrm{H}$ are the width, length, and height of the cavity. It should be noted that the obtained resonance frequencies are for complete rectangular structure and adding slots on the walls lead to shifting the resonances of different mode numbers. In this paper, the final structure is to be used as a sensor. Hence, etching slots on the cavity walls and substrate facilitate merging the sample into the structure and creating a new effective area with different permittivity and permeability based on effective medium theory. Various properties of the located sample in the slots leads to changing the MPA absorption characteristics.

Figure la shows the layout of the cavity unit cell. Fr-4 with thickness $(\mathrm{H})$ of $3.2 \mathrm{~mm}$ has been implemented as a substrate with dielectric constant $\varepsilon_{r}=4.3$ and loss tangent $\tan \delta=0.014$. It has been used with two aspects: first, making the structure low-profile; and second, creating a firm structure that can last for a long time. Two perpendicular slots have been etched on the top wall of the cavity resonator. This layout of slots guarantees the resonating of the cavity facing both TE and TM polarized waves. Moreover, the symmetric structure is a crucial requirement for MPA to be polarization angle insensitive. The design process has been performed using CST Studio 2019 (Version 2019.07). As illustrated in Fig. 1b, a unit cell boundary condition is applied to a unit cell to model the metamaterial structure. Moreover, the Flouque port has been applied to analyse the proposed design under different polarization and incident angles of electromagnetic waves in TE and TM modes.

Based on Eq. (4), in case of a much smaller height $(\mathrm{H})$ of the cavity compared to width (W) and length (L), $T E_{m 0 n}$ modes are resonated in the cavity. Exciting the cavity using slots in the middle of the wall leads to create two electromagnetic zones on two sides of the slot. Consequently, the even modes resonate in the cavity. Moreover, some hybrid modes that are a combination of modes can resonate in the cavity. The dimension of the slot has a significant effect on the resolution of the proposed sensor. Figure 2 shows the absorbance of cavity MPA by varying the slot width (W) and Length (L), respectively. The slot size has a direct effect on matching the input impedance of MPA with intrinsic air impedance. As can be seen in Fig. 2a, widening the slot leads to shifting resonance frequency forward. This is due to decreasing equivalent capacitance of the structure followed by reducing the conductive area. Furthermore, the intensity of electromagnetic waves on two edges of slots is weakened by increasing the distance between them. Therefore, the equivalent capacitance decreases for wider slots due to the lower electrical charge on edges.

Changing the slot length leads to absorption frequency shift, as shown in Fig. 2b. Increasing the slots' length leads to a smaller metallic surface that is one of the effective parameters to increase the equivalent capacitance. However, degrading the edges' length causes a significant drop in the amount of electrical charge on the metallic layer. This phenomenon decreases the unit cell's equivalent capacitance and shifts the resonance frequency upward as a consequence. It should be mentioned that slots' lengths have been kept constant and equal to $l_{s}=46$ to investigate the effect of their width. Similarly, to illustrate the impact of slots' width, their lengths have been kept equal to $w_{s}=8$. 


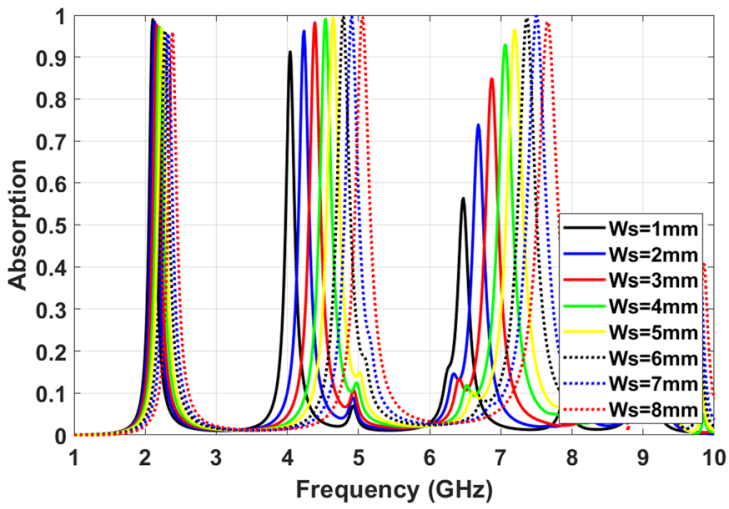

(a)

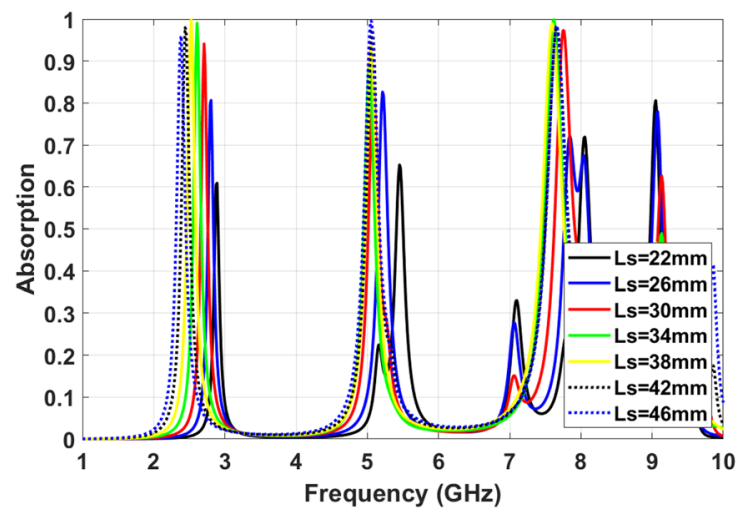

(b)

Figure 2. (a) Effect of changing slots width, (b) Effect of changing slots length.

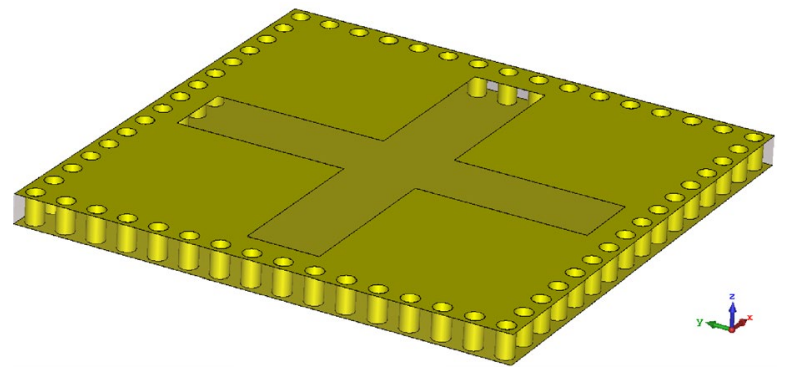

(a)

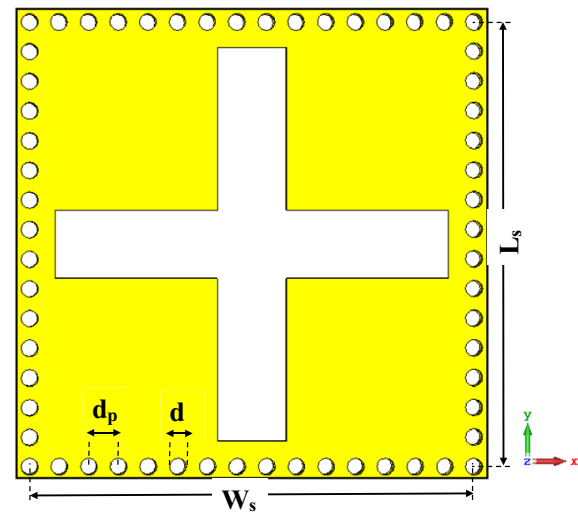

(b)

Figure 3. (a) SIW cavity resonator structure, (b) SIW cavity resonator dimensions.

All resonance modes can be used as a sensing parameter. Nevertheless, the higher mode resonances show more sensitivity due to the more peak points in e-field distribution. The cavity dimensions have been chosen to keep the targeted resonance modes at the commercial frequency spectrum. Both the width (W) and length (L) have been chosen equal to $50 \mathrm{~mm}$ as the cavity's initial dimension. Based on the Eq. (4), the resonance frequencies of first, second, and third modes are at $2.2 \mathrm{GHz}$ and $4.9 \mathrm{GHz} 7.5 \mathrm{GHz}$ for a cavity without slots.

Substrate integrated waveguide structure. The conventional cavity can be modified to a planer and low-profile structure using the SIW technology, as shown in Fig. 3. Placing the metallic via instead of sidewalls of the cavity leads to dropping the propagation of surface waves. Hence, power leakage is reduced, and edge diffraction effects are suppressed. Moreover, using SIW technology improves the concentration of the electromagnetic wave in the cavity. This phenomenon enhances the practicality of proposed MPA in sensing applications. The following relations have to be considered to satisfy the least leakage of electromagnetic waves from SIW cavity walls as well as optimizing the number of hole vias ${ }^{46}$ :

$$
\begin{gathered}
d<\frac{\lambda}{5} \\
d_{p}<2 d
\end{gathered}
$$

where $\mathrm{d}$ is the hole via diameter, $d_{p}$ is center to center distance of to neighbor hole via, see Fig. $3 \mathrm{~b}$. The effects of changing hole's diameter $(d)$ and distance $\left(d_{p}\right)$ considering the Eqs. (5) and (6) have been investigated. The results are shown in Fig. 4a,b. It should be noted that the hole's distance must be chosen by considering the available space to have complete via hole. As can be seen, these two parameters' effects are not significant, if they satisfy Eqs. (5) and (6).

Moreover, Eq. (4) needs to be tailored to obtain SIW cavity resonance. Due to circular shape of hole vias the width (W) and length (L) in Eq. (4) have to be replaced by effective width $\left(W_{\text {eff }}\right)$ and effective length $\left(L_{e f f}\right)$, as calculated below ${ }^{47}$ : 


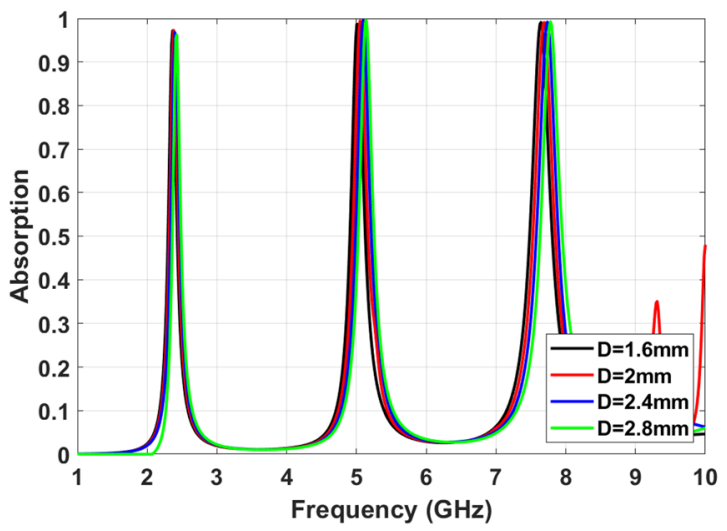

(a)

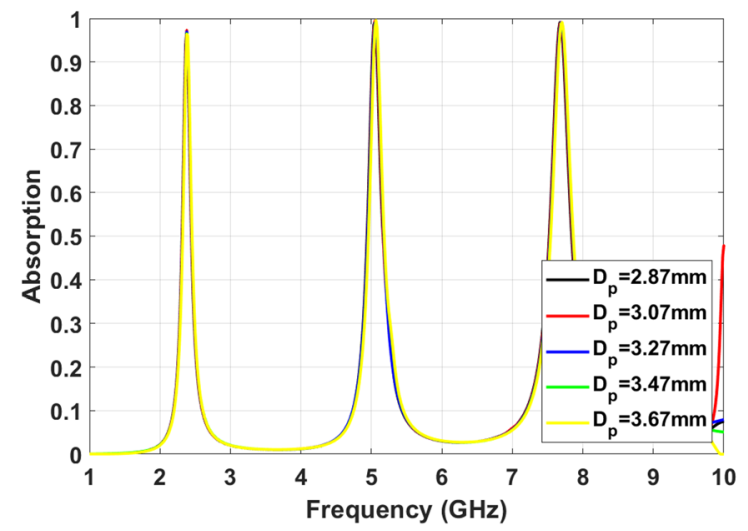

(b)

Figure 4. The effects of via hole parameters on absorption characteristics (a) Hole's diameter, (b) Hole's distance.

\begin{tabular}{|l|l|l|l|l|l|l|l|l|}
\hline Parameter & $\mathbf{L}$ & $\mathbf{W}$ & $\boldsymbol{l}_{\boldsymbol{s}}$ & $\boldsymbol{w}_{\boldsymbol{s}}$ & $\boldsymbol{L}_{\boldsymbol{s}}$ & $\boldsymbol{W}_{\boldsymbol{s}}$ & $\boldsymbol{d}_{\boldsymbol{p}}$ & $\mathbf{d}$ \\
\hline Value & $50 \mathrm{~mm}$ & $50 \mathrm{~mm}$ & $46 \mathrm{~mm}$ & $8 \mathrm{~mm}$ & $51.263 \mathrm{~mm}$ & $51.263 \mathrm{~mm}$ & $3.47 \mathrm{~mm}$ & $2 \mathrm{~mm}$ \\
\hline
\end{tabular}

Table 1. The optimum values of unit cell dimension.

$$
\begin{gathered}
L_{e f f}=L_{c}-1.08 \frac{d^{2}}{d_{p}}+0.1 \frac{d^{2}}{L_{c}} \\
W_{e f f}=W_{c}-1.08 \frac{d^{2}}{d_{p}}+0.1 \frac{d^{2}}{W_{c}}
\end{gathered}
$$

where $L_{c}$ and $W_{c}$ are the length and width of the SIW cavity representing center to center spacing of corner vias. These parameters are calculated using the Eqs. $(7)$ and (8) by considering effective length $\left(L_{\text {eff }}\right)$ and width $\left(W_{\text {eff }}\right)$. To avoid changing cavity resonances after adding the via holes $\left(L_{\text {eff }}=L\right)$ and $\left(W_{\text {eff }}=W\right)$.

\section{MPA simulation results}

Table 1 represents the dimensions of the proposed unit cell to excite the first three modes of SIW cavity at 2.4 $\mathrm{GHz}, 5 \mathrm{GHz}$, and $7.7 \mathrm{GHz}$, based on previously described equations. Before implementing the SIW cavity MPA as a sensor, the proposed MPA's characteristics need to be investigated facing different incident waves, including TE and TM polarized waves. The E-field and $\mathrm{H}$-field distributions of the structure at resonance frequencies facing TE polarized incident waves are showing in Fig. 5, so as to provide better insight into the mechanism of the final structure and explain the resonant mode numbers. The structure's dimension leads to the exciting of $T E_{m n p}$ facing both TE and TM polarized incident waves. Figure 5a,b show the E-field and H-field distributions of $T E_{102}$ mode at $f=2.4 \mathrm{GHz}$. These figures illustrate two electromagnetic zones at the different centers by slot, are out of phase, and have equal amplitude for electric and magnetic fields.

Additional to common modes, one hybrid mode is excited in the proposed SIW cavity at $\mathrm{f}=5 \mathrm{GHz}$. Figure $5 \mathrm{c}$,d show the electric and magnetic field distributions in the unit cell of MPA, respectively. Two completely separated out of phase electromagnetic zones is represented by $T E_{102}$ modes. Moreover, two smaller out of phase electromagnetic zones are created. This mode is a hybrid mode created by combining $T E_{102}$ and $T E_{104}$ modes. Furthermore, $T E_{104}$ mode resonates at $\mathrm{f}=7.7 \mathrm{GHz}$ in the proposed structure. Figure $5 \mathrm{e}$ shows the E-field distribution of structure in this mode. There are four different electric zones with an alternative change in their phases. Also, these four individual zones are seen in H-field distribution; see Fig. $5 f$.

The SIW cavity unit cell and perpendicular slot's dimension leads to similar E-filed and $\mathrm{H}$-field concentration patterns and the other slot (Y-axis) for TM polarized incident waves. It means that $T E_{201}$, hybrid mode of $T E_{201}$ and $T E_{401}$, and $T E_{401}$ modes are excited in the SIW cavity facing TM polarized incident waves. Figure 6 shows the E-field and $\mathrm{H}$-field distribution facing TM polarized incident waves at resonance frequencies.

As shown in Figs. 5 and 6, the waves enter via slots and distribute along the cavity symmetrically. The number of peak points along each $\mathrm{x}$ and $\mathrm{y}$-axis specifies the mode's order for $\mathrm{m}$ and $\mathrm{n}$. However, the E-field's centering around the slots facing different polarized incident waves is common for all mode numbers. This field concentration leads to creating a dipole structure on the vertical or horizontal slot depending on the incident wave polarization. Based on the Maxwell equation, the electric field creates an electrical charge on the metallic surface. The electrical charge interaction on the surface and bottom layer create a magnetic dipole inside the SIW cavity resonator. Two edges of slot and metallic surfaces on top and bottom of the cavity provide the 
(a)

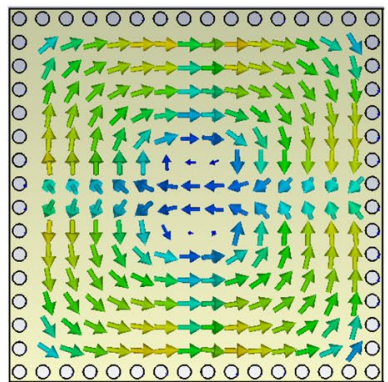

(b) (c)

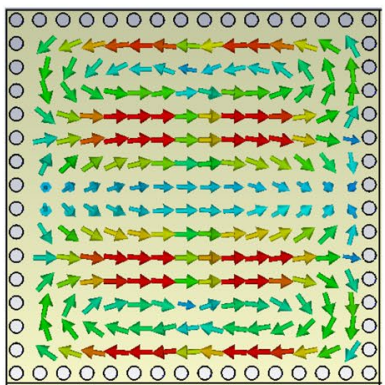

(d) (e)

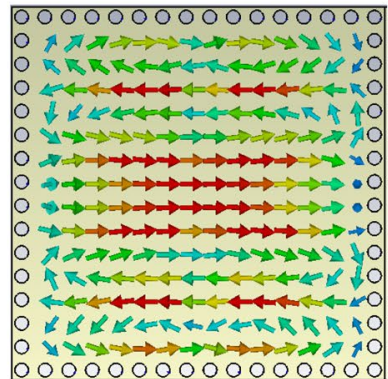

(f)

Figure 5. Electromagnetic fields facing TE polarized incident waves (a) E-field distribution at $f=2.4 \mathrm{GHz}$ $\left(T E_{102}\right),(\mathbf{b}) \mathrm{H}$-field distribution at $f=2.4 \mathrm{GHz}\left(T E_{102}\right)$, (c) E-field distribution at $f=5 \mathrm{GHz}$ (Hybrid mode of $T E_{102}$ and $T E_{104}$ ), $\mathbf{d} \mathrm{H}$-field distribution at $f=5 \mathrm{GHz}$ (Hybrid mode of $T E_{102}$ and $T E_{104}$ ) (e) E-field distribution at $f=7.7 \mathrm{GHz}\left(T E_{104}\right)$, (f) H-field distribution at $f=7.7 \mathrm{GHz}\left(T E_{104}\right)$. This figures have been created by CST Studio 2019 Version 2019.07.

(a)

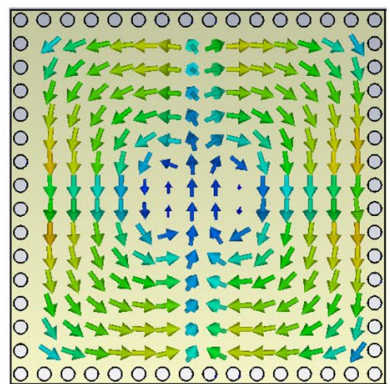

(b) (c)

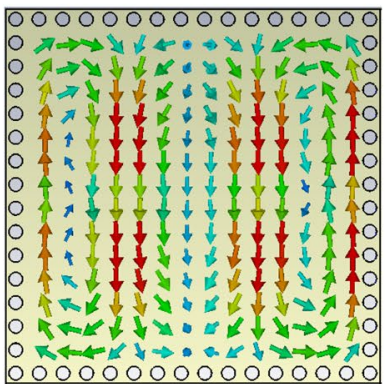

(d) (e)
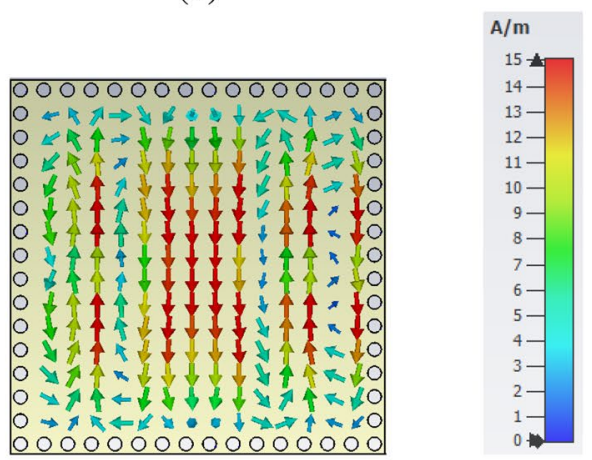

(f)

Figure 6. Electromagnetic fields facing TM polarized incident waves (a) E-field distribution at $f=2.4 \mathrm{GHz}$ $\left(T E_{201}\right)$, b H-field distribution at $f=2.4 \mathrm{GHz}\left(T E_{201}\right)$, (c) E-field distribution at $f=5 \mathrm{GHz}$ (Hybrid mode of $T E_{201}$ and $T E_{401}$ ), (d) H-field distribution at $f=5 \mathrm{GHz}$ (Hybrid mode of $T E_{201}$ and $T E_{401}$ ) (e) E-field distribution at $f=7.7 \mathrm{GHz}\left(T E_{401}\right)$, (f) H-field distribution at $f=7.7 \mathrm{GHz}\left(T E_{104}\right)$. This figures have been created by CST Studio 2019 Version 2019.07. 


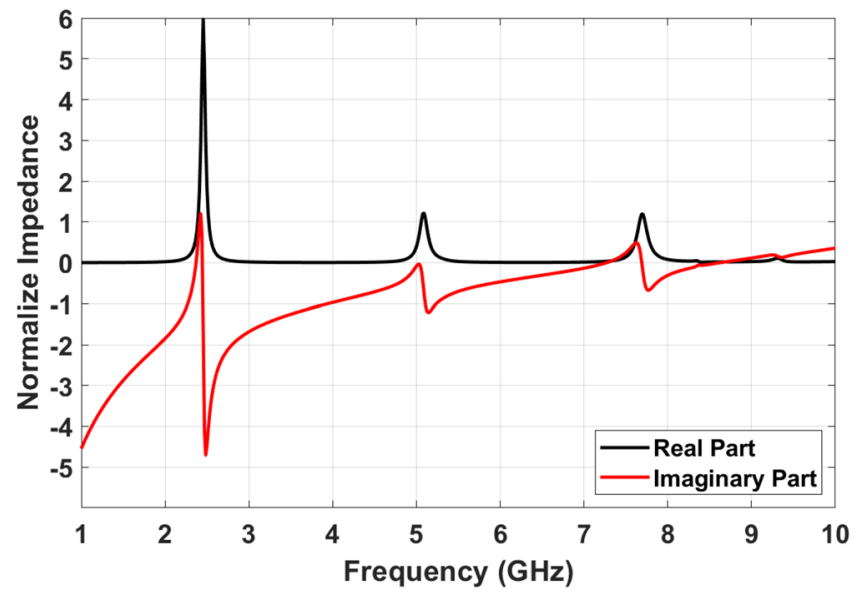

Figure 7. Input impedance of SIW cavity resonator.

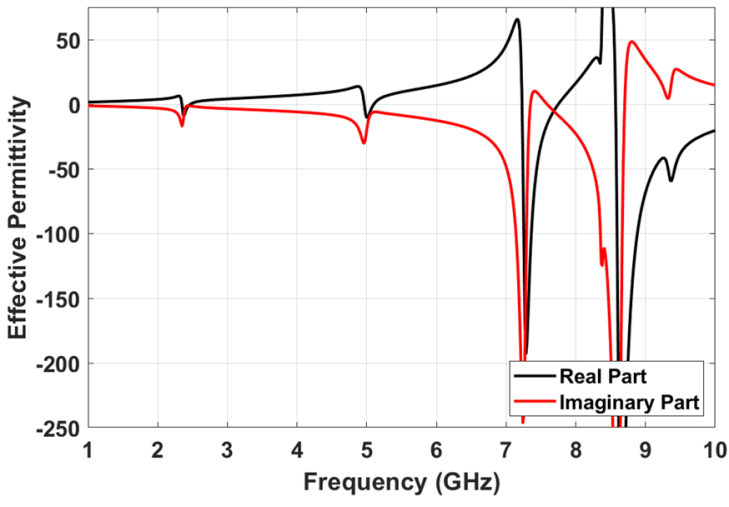

(a)

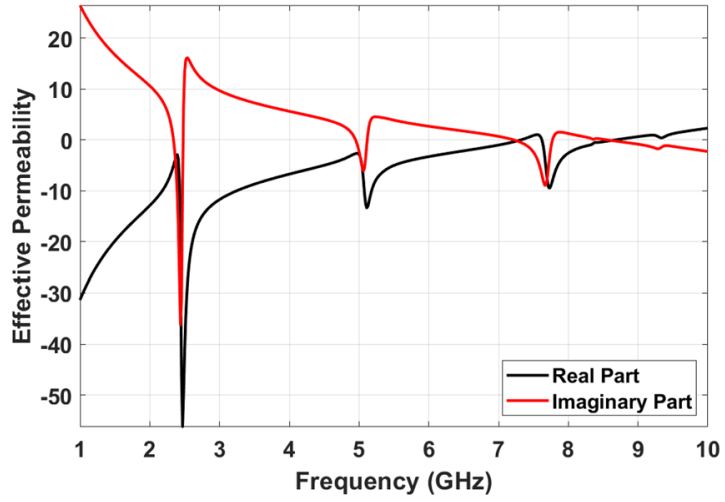

(b)

Figure 8. (a) Effective permittivity, (b) Effective permeability.

structure's capacitive behavior. On the other hand, rotating magnetic fields create surface currents that provide the structure's inductive behavior. The coupling of these structures' properties, which are due to the electric and magnetic responses, leads to a strong local resonance in each cavity resonator mode.

The input impedance of final SIW cavity MPA has been obtained based on Eq. (3) and is shown in Fig. 7. It should be noted that the impedance graph has been normalize to air intrinsic impedance. As mentioned before, ideal absorptivity would be achieved if the real and imaginary parts of input impedance are 1 and 0 , respectively. The input impedances of proposed MPA are $Z_{\text {in } 1}=0.81+j 0.19, Z_{\text {in } 2}=0.91-j 0.09$ and $Z_{\text {in } 3}=1.1-j 0.01$ at $f=2.4 \mathrm{GHz}, f=5.05 \mathrm{GHz}$ and $f=7.65 \mathrm{GHz}$, respectively. The small difference between $Z_{\text {in }}$ at resonance frequencies and $Z_{0}$ confirm the near unity absorption based on Eq. (1). The Q-factors are 26.4, 34.8 and 36.61 at resonance frequencies in order. Moreover, effective permeability and permittivity are two other important parameters to show the metamaterial behaviour. These parameters are calculated using the refractive index of structure $^{44,48}$ based on Eq. (9):

$$
n_{e f f}(\omega)= \pm\left(\frac{1}{k L}\right) \arccos \left(\frac{1-S_{11}^{2}+S_{21}^{2}}{2 S_{21}}\right)
$$

Where $\mathrm{k}$ and $\mathrm{L}$ are the wave wavelength and the unit cell size, respectively. By calculating the MPA impedance and effective refractive index, permeability and permittivity are defined in Eqs. (10) and (11), respectively. As can be seen in Fig. 8a,b, both effective permittivity and permeability are negative around resonance frequency $\left(\varepsilon_{\text {eff }}<0\right.$ and $\left.\mu_{\text {eff }}<0\right)$.

$$
\begin{aligned}
& \mu_{\text {eff }}(\omega)=n_{\text {eff }}(\omega) Z_{\text {eff }}(\omega) \\
& \varepsilon_{e f f}(\omega)=n_{\text {eff }}(\omega) / Z_{\text {eff }}(\omega)
\end{aligned}
$$

The insensitivity of absorption characteristics facing various incident waves is an important parameter due to different polarization and incident angles. Some reported designs show a perfect insensitivity facing different 


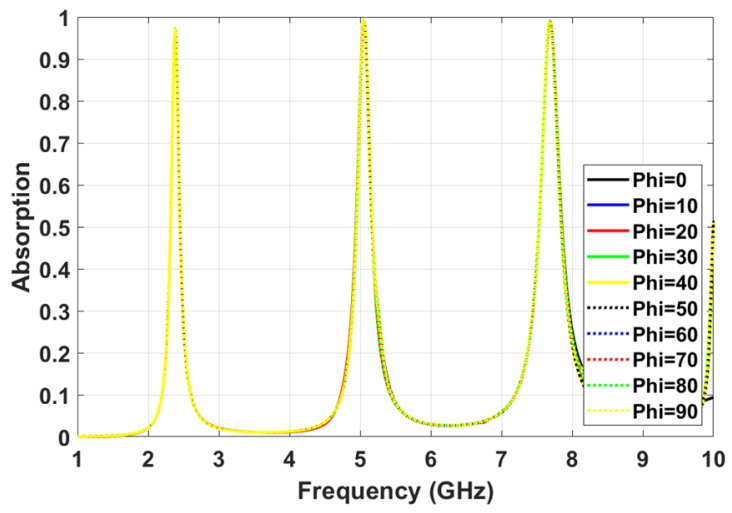

(a)

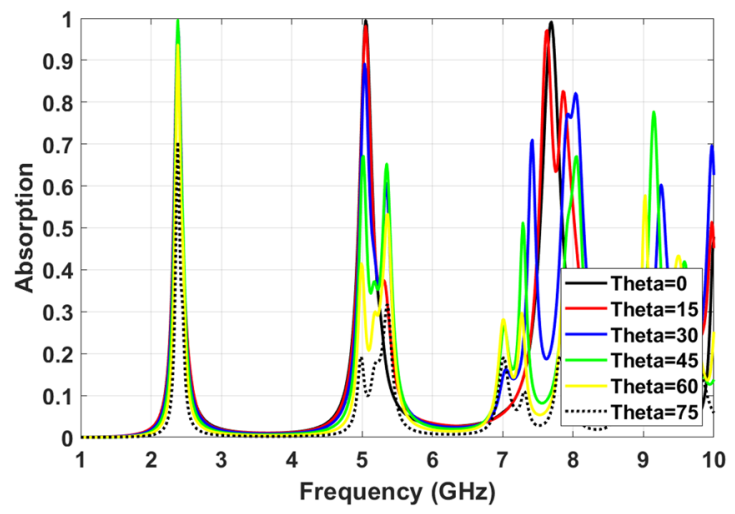

(c)

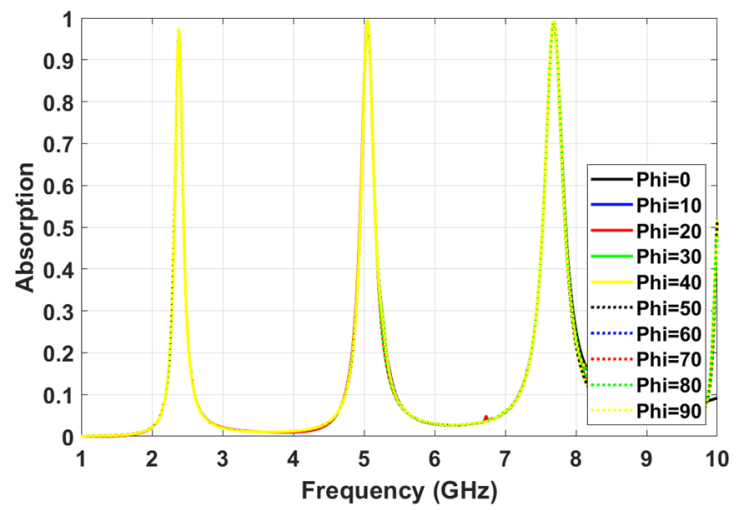

(b)

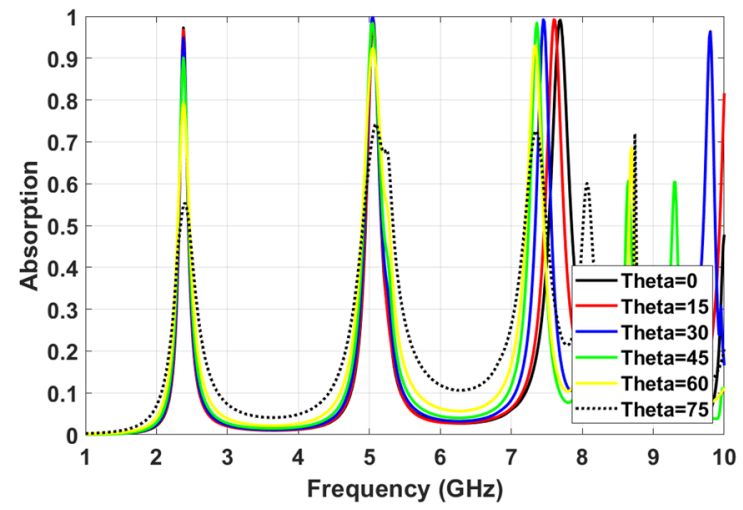

(d)

Figure 9. Angle insensitivity (a) Different polarization angles for TE waves, (b) Different polarization angles for TM waves, (c) Different incident angles for TE waves, (d) Different incident angles for TM waves.

polarization and incident angles for a broad spectrum ${ }^{49,50}$. However, having a high Q-factor followed by sharp resonance is crucial for sensing applications. The proposed SIW cavity MPA shows perfect insensitivity for changing polarization angles from $\phi=0$ to $\phi=90$ because of the symmetric shape along with high Q-factor at all resonance frequencies. Fig. 9a,b illustrate that the absorption characteristic remains steady for all polarization angles for TE and TM polarized modes. Also, It can be seen that the proposed structure is the polarization angle insensitive for all the mode numbers.

On the other hand, the insensitivity of absorption characteristics facing different incident angle shows various behavior for different model numbers. As Fig. $9 \mathrm{c}$ shows, for $T E_{102}$ mode $(\mathrm{f}=2.4 \mathrm{GHz})$, absorption ratio remains above $80 \%$ by increasing the incident angles up to $\theta=60^{\circ}$. Furthermore, the resonance frequency shows almost no shift by increasing the incident angles. Moreover, for hybrid mode ( $\mathrm{f}=5 \mathrm{GHz}$ ), the absorption ratio is more than $60 \%$ for incident angles to $\theta=45^{\circ}$. Also, the absorption frequency shifts are negligible. Whereas, for $T E_{104}$ mode $(\mathrm{f}=7.7 \mathrm{GHz})$, the absorption ratio stays higher than $65 \%$ incident angles to $\theta=45^{\circ}$, while the absorption frequency shifts from $7.7 \mathrm{GHz}$ to $8 \mathrm{GHz}$ for this range of incident angle.

Facing TM polarized incident waves, the absorption stability for $T E_{102}(\mathrm{f}=2.4 \mathrm{GHz})$ is slightly less than $\mathrm{TE}$ mode. However, the absorption ratio is higher than $80 \%$ at incident wave angles up to $\theta=60^{\circ}$ that is reasonable absorption insensitivity. Nevertheless, for hybrid mode $(\mathrm{f}=5 \mathrm{GHz})$, the absorption characteristics show better insensitivity facing TM waves compared to TE ones. The absorption ratios of hybrid mode at $\theta=60^{\circ}$ and $\theta=75^{\circ}$ are $92 \%$ and $74 \%$, respectively. Meanwhile, the absorption frequency shows a negligible shift and is fairly stable at high incident angles up to $\theta=75^{\circ}$. As it can be seen in Fig. $9 \mathrm{~d}$ the absorption ratios of $T E_{401}$ at $\theta=60^{\circ}$ and $\theta=75^{\circ}$ are $93 \%$ and $71 \%$, respectively. However, the absorption frequency shifts by $400 \mathrm{MHz}$. Based on the represented results in Fig. 9c,d, the insensitivity for different incident angles is significant for both TE and TM modes at the first and second resonances. This is due to the strong magnetic response of the cavity resonator.

The soil moisture remote sensing setup can be implemented using controllable or random electromagnetic signals. In controlled scenarios, the position of the transmitter, receiver, and sensor are chosen. Hence, the incident angle can be easily set. However, the sensor should be insensitive enough for changing polarization and incident angles to handle implementation errors. On the other hand, random ambient electromagnetic signals are other candidates to be used in sensing applications. In this case, the sensor must be insensitive for changing incident and polarization angles. Therefore, the first two resonances are appropriate for both controlled sensing scenarios and uncontrolled sensing. On the other hand, the third resonance is not suitable for uncontrolled sensing scenarios due to the possibility of false sensor reading for the higher incident angle. However, the third mode's high resolution can be implemented to sense multiple soil parameters such as salinity and nutrition simultaneously. 


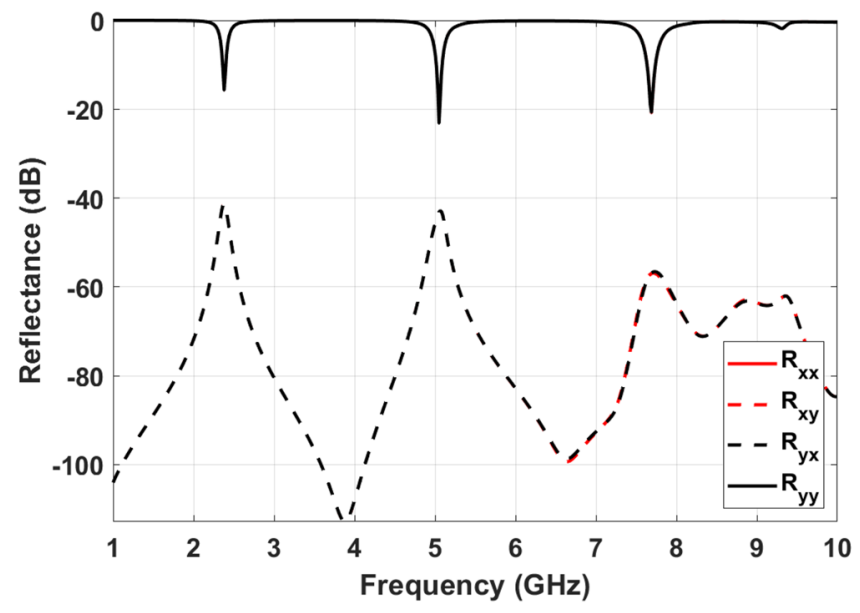

Figure 10. Co- and cross-polarization reflection.

Converting the polarization instead of absorbing the incident signals can lead to sensor inaccuracy. To avoid this issue, polarization conversion ratio (PCR) has to be minimum. In more detail, the reflected waves from MPA structure consist co-polarized and cross-polarized components. Co-polarization reflection coefficients can be in $\mathrm{x}$ and $\mathrm{y}$ directions that are defined as $R_{x x}=\left|E_{r x}\right| /\left|E_{t x}\right|$ and $R_{y y}=\left|E_{r y}\right| /\left|E_{t y}\right|$. Furthermore, cross-polarization reflection coefficient are determined as $R_{x y}=\left|E_{r x}\right| /\left|E_{t y}\right|$ and $R_{y x}=\left|E_{r y}\right| /\left|E_{t x}\right|$. Where $E_{t x}$ and $E_{t y}$ are defined as the X-polarized and y-polarized components of transmitted waves. In addition, $E_{r x}$ and $E_{r y}$ are components of reflected waves. Considering described coefficients, PCR for TE and TM modes are calculated based on Eqs. (12) and (13).

$$
\begin{aligned}
P C R_{T E} & =\frac{R_{y x}^{2}}{R_{y y}^{2}+R_{y x}^{2}} \\
P C R_{T M} & =\frac{R_{x y}^{2}}{R_{x x}^{2}+R_{x y}^{2}}
\end{aligned}
$$

Figure 10 shows the reluctance coefficients of proposed MPA, which are similar in $\mathrm{x}$ and $\mathrm{y}$-direction due to the symmetrical structure. As can be seen, cross-polarization components are negligible even in resonance frequencies. The PCR of the structure facing TE and TM polarized incident waves are $0.0027,0.0099$, and 0.00024 at first, second, and third resonance frequencies. Near zero PCR illustrate the absorption capability of the proposed structure without polarization conversion.

Channeling to create effective medium. As mentioned before, adding an air gap as a sample holder impacts the fabrication complexity and practicality of structure in real-world scenarios. In this paper, perpendicular channels between the slots have been etched in the substrate. The sample can be easily placed in these channels, even for a large structure. Moreover, there is no need to add an extra layer to place the sample in this approach. Figure 11a shows the channeled unit cell of SIW cavity MPA. The combination of dielectric and soil in channels creates a new medium that is represented by effective permittivity and permeability $\left(\varepsilon_{\text {eff }}\right.$ and $\left.\mu_{\text {eff }}\right)$. The slot's rectangular layout leads to maximum soil sample interaction and electric field on slot's edges. The symmetric pattern of the final structure leads to significant insensitivity facing different polarization and incident angles. Meanwhile, this shape of channels facilitates the maximum electromagnetic waves passing through the sample that leads to a higher resolution of the proposed sensor.

It is noteworthy that more volume of the sample leads to more shifts in absorption frequency. Hence, these channel's depth is an important parameter to achieve the best performance that needs to be investigated with two aspects. One is the sensitivity of MPA that is increased by deepening the channels. The second consideration is the rigidity of structure in practical applications that decreases for deeper channels. Figure 11b shows the shift of absorption frequency by changing the soil's water content from $0 \%$ to $30 \%$ for different channel depths. As can be seen, the sample's effect on absorption characteristics increases for deeper channels. This is due to the bigger change in effective permittivity and permeability. It is also obvious from Fig. $9 \mathrm{~b}$ that frequency shift increases at higher resonance modes. In the proposed MPA structure, the depth of the channels has been chosen as $d=2.8$ $\mathrm{mm}$. The sensitivity is slightly compromised by not deepening the channel more to have a solid structure for real-world applications. 


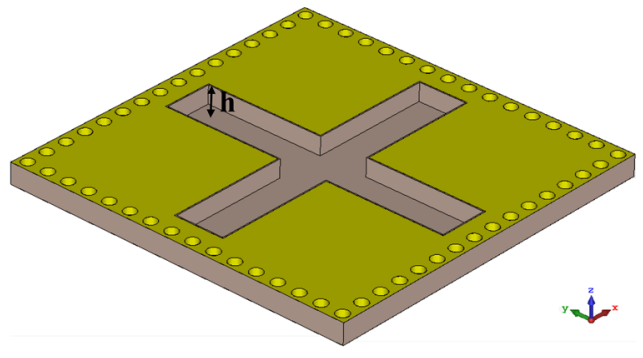

(a)

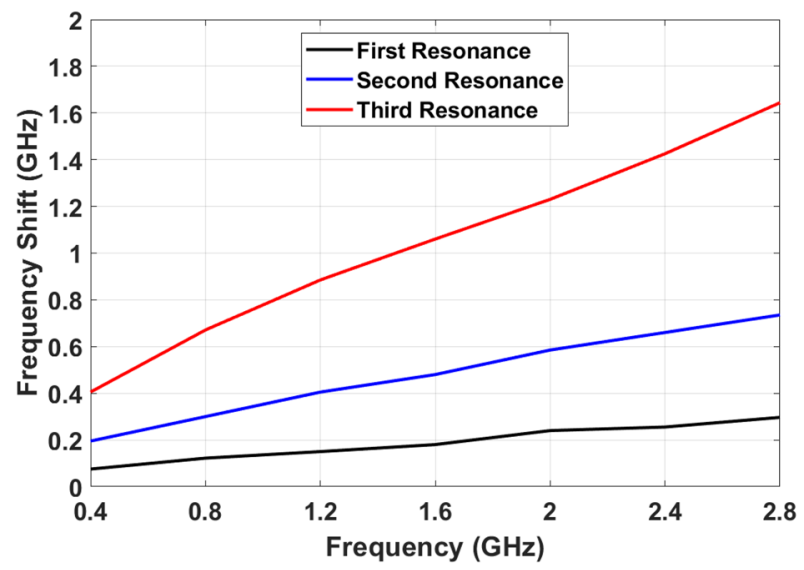

(b)

Figure 11. (a) Final unit cell after etching the channels, (b) The frequency shift by changing the soil moisture content from $0 \%$ to $30 \%$ for different depth of channels.

\begin{tabular}{|c|c|c|c|}
\hline Soil type & Moisture level & Dielectric constant & $\tan \delta$ \\
\hline \multirow{7}{*}{ Sand } & $0 \%$ & 3.0 & 0.033 \\
\hline & $5 \%$ & 3.9 & 0.153 \\
\hline & $10 \%$ & 5.3 & 0.2 .7 \\
\hline & $15 \%$ & 7.2 & 0.236 \\
\hline & $20 \%$ & 9.9 & 0.232 \\
\hline & $25 \%$ & 13.2 & 0.227 \\
\hline & $30 \%$ & 16.9 & 0.224 \\
\hline \multirow{9}{*}{ Loamy } & $0 \%$ & 3.0 & 0.033 \\
\hline & $5 \%$ & 3.8 & 0.131 \\
\hline & $10 \%$ & 4.8 & 0.187 \\
\hline & $15 \%$ & 6.3 & 0.222 \\
\hline & $20 \%$ & 8.5 & 0.235 \\
\hline & $25 \%$ & 11.6 & 0.215 \\
\hline & $30 \%$ & 15.4 & 0.207 \\
\hline & $35 \%$ & 19.2 & 0.198 \\
\hline & $40 \%$ & 23 & 0.191 \\
\hline
\end{tabular}

Table 2. The characteristics of various types of soil with different levels of moisture.

\section{Soil properties with different level of moisture}

In this paper, sensing different levels of soil moisture has been targeted. Thus, soil properties with varying water content levels must be investigated prior to adding them to the structure. Permittivity $\left(\varepsilon_{\text {soil }}\right)$ and loss tangent $(\tan \delta)$ are important characteristics of under test material in sensing process. The behaviour of material in electric field is described as Eq. $(14)^{45}$ :

$$
\varepsilon_{r}=\varepsilon_{r}^{\prime}+j \varepsilon_{r}^{\prime \prime}
$$

where $\varepsilon_{r}^{\prime}$ is the dielectric constant that represent the capability of the storing electric field, while $\varepsilon_{r}^{\prime \prime}$ is the loss factor that shows the ratio of electric field loss in the material. Using these parameters, the loss tangent is defined according to the Eq. (15):

$$
\tan \delta=\frac{\varepsilon_{r}^{\prime \prime}}{\varepsilon_{r}^{\prime}}
$$

$\mathrm{In}^{51}$, the properties of different soil types with various moisture levels have been investigated at different spectrums. Considering Eqs. (14) and (15), sand, loam, and clay soil characteristics with various moisture levels at $4 \mathrm{GHz}$ spectrum have been represented in Table 2. It should be noted that the soil properties at $2.4 \mathrm{GHz}$ and 5 $\mathrm{GHz}$ can be estimated by their values at $4 \mathrm{GHz}$ due to minor changes in properties in this range of frequency ${ }^{52}$. 


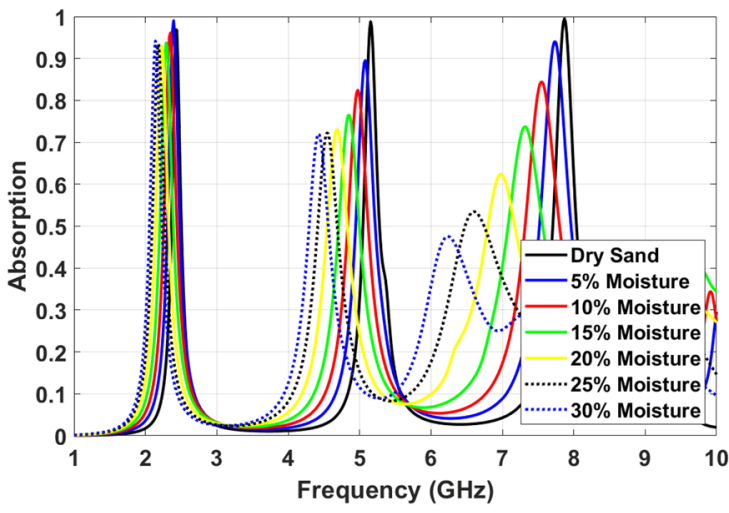

(a)

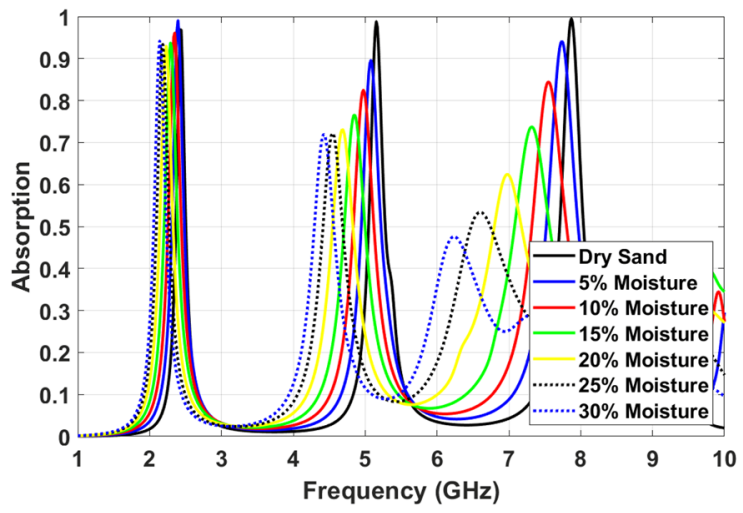

(b)

Figure 12. Sensing efficiency of the proposed MPA for different moisture levels in sand soil (a) Facing TE polarized incident waves, (b) Facing TM polarized incident waves.

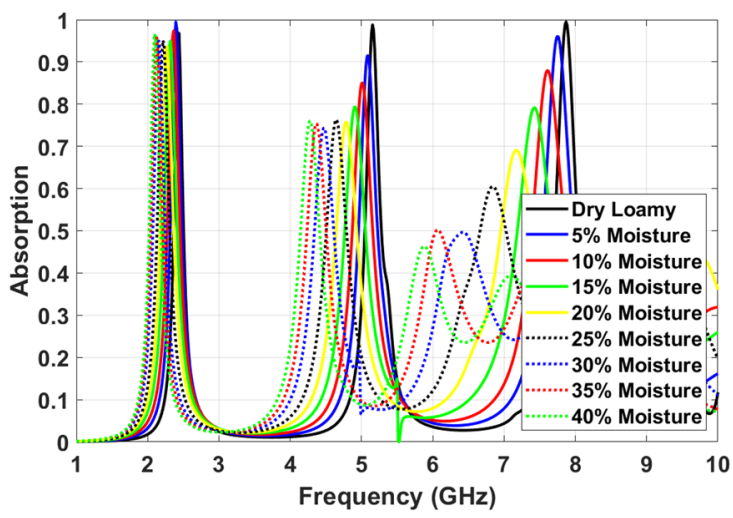

(a)

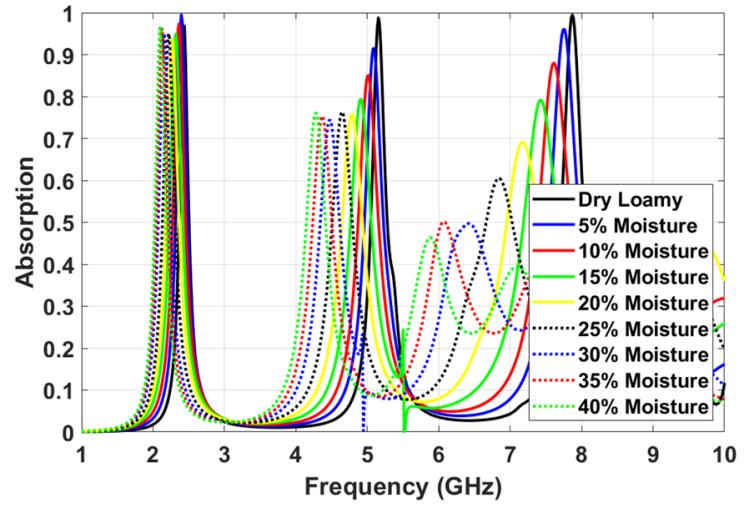

(b)

Figure 13. Sensing efficiency of the proposed MPA for different moisture levels in Loamy soil (a) Facing TE polarized incident waves, (b) Facing TM polarized incident waves.

\section{Sensing capability of proposed sensor}

The absorption ratio and frequency of SIW cavity MPA show different changes for various depths of channels at $\mathrm{f}=2.4 \mathrm{GHz}, \mathrm{f}=5 \mathrm{GHz}$, and $\mathrm{f}=7.7 \mathrm{GHz}$. Figure $12 \mathrm{a}$ shows these changes facing TE polarized incident waves. In this regard, the proposed structure's sensing capability needs to be investigated individually for each frequency. The first resonance frequency $\left(T E_{101}\right)$ shifts from 2.4 to $2.1 \mathrm{GHz}$ when soil moisture content increases from 0 to $30 \%$. This shift in absorption frequency can be considered as $10 \mathrm{MHz}$ per one percentage of moisture content. The absorption ratio varies as the soil moisture changes. This can then be considered as an additional parameter when sensing soil moisture. However, for this mode, the absorption change is negligible within the $300 \mathrm{MHz}$ shift of frequency.

At $\mathrm{f}=5 \mathrm{GHz}$ ( $T E_{103}$ mode), the absorption frequency shifts $700 \mathrm{MHz}$ downward for $30 \%$ change in soil moisture, which translates to $23.3 \mathrm{MHz}$ per one percentage of water content. Furthermore, the absorption ratio of the proposed structure at the second absorption band decreases almost linearly from 99.8 to $82 \%$ when moisture changes $30 \%$. At $\mathrm{f}=7.8 \mathrm{GHz}$ (TE 105 mode), the frequency shifts $1.8 \mathrm{GHz}$ and absorption ratio drops from 99.9 to $48 \%$. These parameters can be translated to a $60 \mathrm{MHz}$ frequency shift and $1.8 \%$ percentage of absorption ratio per one percent of soil moisture level. Therefore, the fifth mode shows better sensitivity to the level of water content in the soil. Due to the dimension and the unity ratio of width and length of the proposed cavity-based MPA, similar behavior can be seen facing TM polarized incident waves, see Fig. $12 \mathrm{~b}$. In this case, $T E_{101}, T E_{101}$ and $T E_{101}$ modes are excited at $\mathrm{f}=2.4 \mathrm{GHz}, \mathrm{f}=5 \mathrm{GHz}$ and $\mathrm{f}=7.8 \mathrm{GHz}$, respectively.

The proposed structure can be implemented to sense moisture content in different types of soil. Figure 13a,b shows the absorption characteristics of SIW cavity MPA by adding Loamy soil with different levels of moisture in channels facing TE and TM polarized incident waves, respectively. As can be seen in these figures, the absorption characteristics show similar behavior for different soil types. It means the absorption ratio drops by increasing the soil moisture level, and the absorption frequency shifts downward. Moreover, the sensitivity of MPA increases for higher resonance order.

Facing TE polarized incident waves, at the first resonance frequency, the absorption ratio is almost unchanged and higher than $95 \%$ for various levels of moisture, as shown in Fig. 13a. However, the absorption frequency shift 


\begin{tabular}{|c|c|c|c|c|c|c|c|c|c|}
\hline \multirow[b]{2}{*}{ Advantages } & \multicolumn{9}{|l|}{ Category } \\
\hline & Sensor type & Accuracy & Resolution & Longevity & $\begin{array}{l}\text { Measurement } \\
\text { zone }\end{array}$ & $\begin{array}{l}\text { Large scale } \\
\text { deployment }\end{array}$ & Cost & Maintenance & Connection \\
\hline \multirow{4}{*}{$\begin{array}{l}\text { Commercial } \\
\text { Sensor }\end{array}$} & TDR/TDT & Excellent & Excellent & 20 years & $<1 \mathrm{~cm}$ from sensor & $\begin{array}{l}\text { Hard and Expen- } \\
\text { sive }\end{array}$ & High & Not Required & Required \\
\hline & Capacitance/FDR & Satisfactory & Good & 2 to 5 years & $\begin{array}{l}<1 \mathrm{~cm} \text { from } \\
\text { sensor }\end{array}$ & Expensive & Low & Calibration & Required \\
\hline & Standing Wave & Good & Excellent & 20 years & $\begin{array}{l}2 \mathrm{~cm} \text { from central } \\
\text { needle }\end{array}$ & Expensive & Moderate & Calibration & Required \\
\hline & Neutron Prob & Excellent & Excellent & 20 years & $\begin{array}{l}30 \mathrm{~cm} \text { diameter } \\
\text { sphere }\end{array}$ & Expensive & High & Calibration & Required \\
\hline \multirow{3}{*}{ Scientific Sensors } & $\begin{array}{l}\text { Metamaterial } \\
\text { Filter }^{53-55}\end{array}$ & Excellent & Satisfactory & $\begin{array}{l}\text { Long(Depends on } \\
\text { Material) }\end{array}$ & Small & Impossible & Low & Not Required & Required \\
\hline & $\begin{array}{l}\text { Multi-layer MPA } \\
\text { with PDMS }\end{array}$ & Excellent & Satisfactory & $\begin{array}{l}\text { Long(Depends on } \\
\text { Material) }\end{array}$ & Medium & Impossible & Moderate & Not Required & Required \\
\hline & $\begin{array}{l}\text { Multi-layer MPA } \\
\text { Air-Gap } \\
\end{array}$ & Excellent & Satisfactory & $\begin{array}{l}\text { Long(Depends on } \\
\text { Material) }\end{array}$ & Medium & Impossible & Low & Not Required & Required \\
\hline Proposed Sensor & Cavity-based MPA & Excellent & Excellent & Life-Time & Big & $\begin{array}{l}\text { Easy and Inex- } \\
\text { pensive }\end{array}$ & Low & Not Required & Not Required \\
\hline
\end{tabular}

Table 3. Comparing different types of soil moisture sensor based on various methods.

from 2.4 to $2.1 \mathrm{GHz}$ when the soil moisture changes from 0 to $40 \%$. It means almost $7.5 \mathrm{MHz}$ per percentage of soil moisture. At the second resonance frequency, the absorption frequency shifts from 5.13 to $4.23 \mathrm{GHz}$ by adding Loamy soil moisture levels up to $40 \%$. This shift means $22.5 \mathrm{MHz}$ change per percentage of soil moisture. Besides, the absorption ratio falls off from 99 to $76 \%$. Similar to sensing the sand moisture level, the third absorption frequency shows the highest sensitivity. Dropping the absorption ration from 99.9 to $46 \%$ and shifting the absorption frequency from 7.9 to $5.8 \mathrm{GHz}$ for changing soil moisture in the range of $0 \%$ and $40 \%$ provides a highly sensitive low-profile sensor. It should be noted that the sensing capability of the structure facing TM polarized incident waves is similar to TE mode, as can be seen in Fig. $13 \mathrm{~b}$.

Different methods have been implemented to design highly sensitive and robust soil moisture sensors. Table 3 has been provided to compare the capability of the proposed structure, commercial, and previously reported scientific sensors. This comparison covers various parameters, such as accuracy, resolution, longevity, cost, and maintenance requirement. As can be seen, the proposed sensor offers high resolution and the highest life expectancy. Also, unlike commercial sensors, the proposed structure can be fabricated and implemented at a much lower cost. In addition to the accuracy and resolution, deployment capability on a large scale is a crucial requirement in modern agriculture. Commercial sensors consist of a probe that interacts with the environment and an analyser that processes the probe's output into presentable data. For large scale deployment, the sensor's analyser has to be connected to a data transmission network. Implementing a compatible transmission network can be a challenging and expensive process requiring extra infrastructure. The proposed structure is able to perform wirelessly with no need for an attached analyser section. The analyser section includes a signal transmitter, a signal receiver, and a signal processor that can be placed at a distance from the sensors. The concept of wireless sensing with one data analyser unit for all sensing scenarios removes the need for a complicated data transmission network.

Radio frequency sensors found in scientific literature ${ }^{27,28,39,40,42,53-57}$ have been designed to sense solid or liquid analytes. The common consideration in design procedure among all reported structures is creating a specific place to add analyte into the sensor. Taking advantage of radio frequency behaviour facing different mediums, changing transmission, or reflection coefficients by introducing analyte is considered as a sensing parameter. The reported structures are low-profile and show reasonable sensitivity and longevity. However, these sensors are not suitable for remote sensing scenarios as they need a physical connection to add analyte or transmit RF signals through the structure. The proposed structure in this paper has been compared with reported sensors in the literature that used various methods in Table 4 . The performance of sensors is defined by sensitivity. Various equations have been used to calculate this parameter. Herein, the average sensitivity of sensor $\left(S_{\text {ave }}=\left(\Delta f / f_{0}\right) /\left(\Delta \varepsilon / \varepsilon_{\text {eff }}\right)\right.$ has been considered to unify the sensing capability targeting different analytes ${ }^{58}$. Where $\varepsilon_{\text {eff }}$ is the maximum of $\varepsilon_{r}$ considering the variation in analyte's concentration. As shown in Table 4, the sensitivity of the proposed sensor shows significant improvement compared to previously reported structures. It should be noted that the Table 4 comparisons are based upon simulation result to provide a fair comparison.

\section{Fabrication and measurement}

To prove the design procedure and the simulation results, the final SIW cavity MPA has been fabricated in a $6 \times 6$ array structure that is shown in Fig. 14a. The plus shape slots have been etched on the copper layer on top of the FR-4 substrate. Then, the via holes and channels have been created in the structure. Two horn antennas are located one meter away from MPA to satisfy the far-field distance (more than $10 \lambda$ ). One of the antennas works as a transmitter, and the other one works as a receiver. Absorption characteristics for different polarization angles are measured by rotating reference antennas around the horizontal axis simultaneously. On the other hand, to change the incident angle, horn antennas are moved on a half-circle with a radius of one meter in opposite directions. Figure $14 \mathrm{~b}$ shows the measurement setup. Prior to performing the measurements, the setup must 


\begin{tabular}{|c|c|c|c|c|c|c|c|c|c|c|}
\hline Reference & Method & $f_{0} \mathrm{GHz}$ & $\Delta f$ & $\min \varepsilon_{r}$ & $\max \varepsilon_{r}$ & Sensitivity & Analyte & Concentration & Wireless sensing & $\begin{array}{l}\text { Changing } \\
\text { analyte }\end{array}$ \\
\hline 42 & SIW Antenna & 5.09 & $180 \mathrm{MHz}$ & 5.22 & 75 & 3.8 & Ethanol & $5-100 \%$ & No & Required \\
\hline 53 & SRR filter & 1.895 & $110 \mathrm{MHz}$ & 8.4 & 79 & 6.5 & Ethanol & $0-100 \%$ & No & Required \\
\hline 54 & CSRR filter & 1.73 & $400 \mathrm{MHz}$ & 9.7 & 79 & 26.3 & Ethanol & $0-100 \%$ & No & Required \\
\hline 55 & Resonator filter & 10.94 & $1.04 \mathrm{GHz}$ & 4 & 44 & 10.45 & Ethanol & $0-100 \%$ & No & Required \\
\hline 40 & SIW Antenna & 4.4 & $400 \mathrm{MHz}$ & 5.4 & 75 & 9.8 & Ethanol & $0-100 \%$ & No & Required \\
\hline 56 & MTM-based Filter & 0.89 & $60 \mathrm{MHz}$ & 27.86 & 80 & 11.75 & Ethanol & $10-100 \%$ & No & Required \\
\hline 39 & SIW Filter & 1.59 & $40 \mathrm{MHz}$ & 21 & 79 & 3.42 & Acetone & $0-100 \%$ & No & Required \\
\hline 27 & $\begin{array}{l}\text { MPA with PDMS } \\
\text { layer }\end{array}$ & 9.49 & $1.09 \mathrm{GHz}$ & 4.5 & 68 & 12.3 & Ethanol & $0-100 \%$ & No & Required \\
\hline 57 & $\begin{array}{l}\text { MPA with PDMS } \\
\text { layer }\end{array}$ & 4.1 & $400 \mathrm{MHz}$ & 23 & 66 & 14.8 & Ethanol & $0-100 \%$ & No & Required \\
\hline 28 & MPA with air gap & 7.31 & $410 \mathrm{MHz}$ & 2.95 & 3.38 & 44.1 & Mass Fraction & $0-100 \%$ & Yes & Required \\
\hline $\begin{array}{l}\text { Proposed } \\
\text { Structure }\end{array}$ & SIW Cavity MPA & $\begin{array}{l}2.4,5.1 \\
7.7\end{array}$ & $\begin{array}{l}300 \mathrm{MHz}, 700 \\
\mathrm{MHz} \\
1.8 \mathrm{GHz}\end{array}$ & 3 & 16.9 & $\begin{array}{l}50.66,55.62 \\
94.74\end{array}$ & Soil Moisture & $0-30 \%$ & Yes & $\begin{array}{l}\text { Not } \\
\text { Required }\end{array}$ \\
\hline
\end{tabular}

Table 4. Some reported MPAs for removing undesired signals applications.

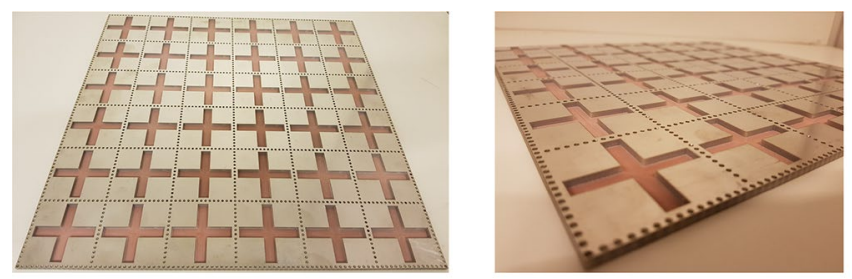

(a)

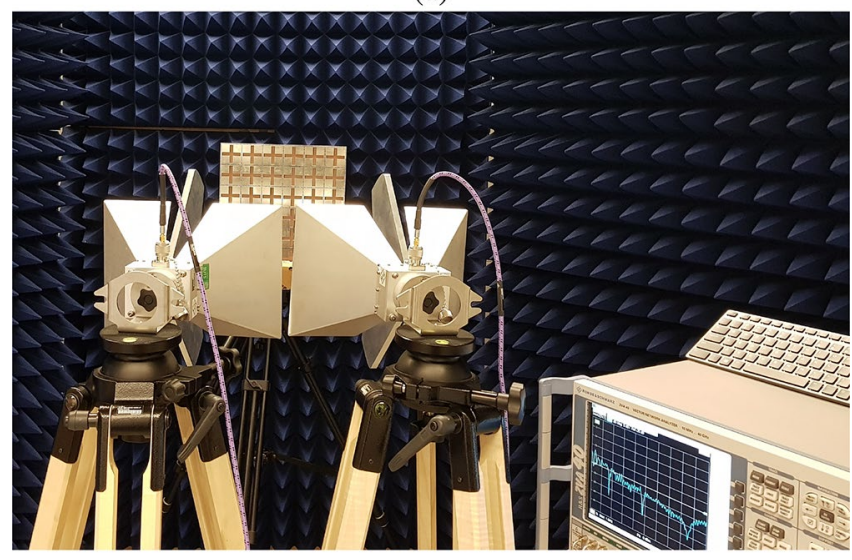

(b)

Figure 14. (a) Fabricated $6 \times 6 \mathrm{MPA}$, (b) Implemented measurement setup.

be calibrated to specify the transmission coefficient's base level between two antennas. In this regard, a metal plate with the same MPA size is placed instead of the final structure. These results are used to reduce the noise of measured characteristics.

Figure 15a,b show the measured absorption at different incident angles from $\theta=0^{\circ}$ to $\theta=60^{\circ}$ under TE and TM polarized radiated electromagnetic waves, respectively. Moreover, Fig. $15 \mathrm{c}$ shows the absorption ratio's measurement results at different polarization angles. The almost unchanged absorption ratio confirms the polarization angle insensitivity of the proposed MPA structure due to the symmetric resonator shape. Fig. 15 implies that measured absorption characteristics of the final structure have a significant correlation with simulation results.

After validating MPA's performance with empty channels, sandy soil with different moisture content has been added to the structure. Different soil moisture levels have been achieved based on the "Soil survey standard test method soil moisture content" 59 that has been released by the Department of Sustainable Natural Resources NSW Australia. The sand has been put in the oven initially to dry the sand. Then, the water has been added to provide sand with $5 \%, 10 \%, 15 \%, 20 \%, 25 \%$, and $30 \%$ soil moisture levels. The soil moisture as a percentage of dry soil weight has been calculated according to the Eq. $(16)^{59}$. 


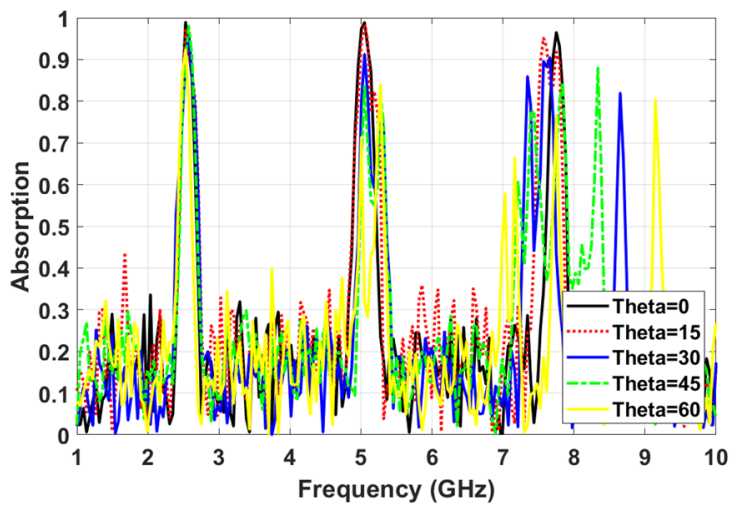

(a)

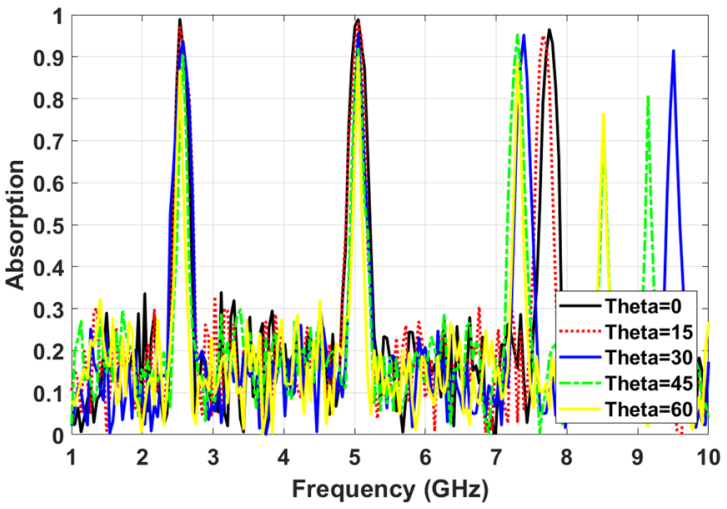

(b)

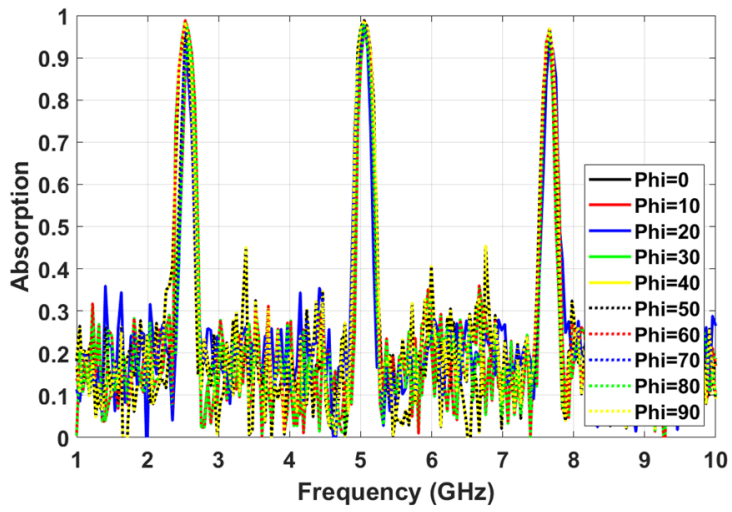

(c)

Figure 15. Angle insensitivity measurement results (a) Different incident angles for TE waves, (b) Different incident angles for TM waves, (c) Different polarization angles.

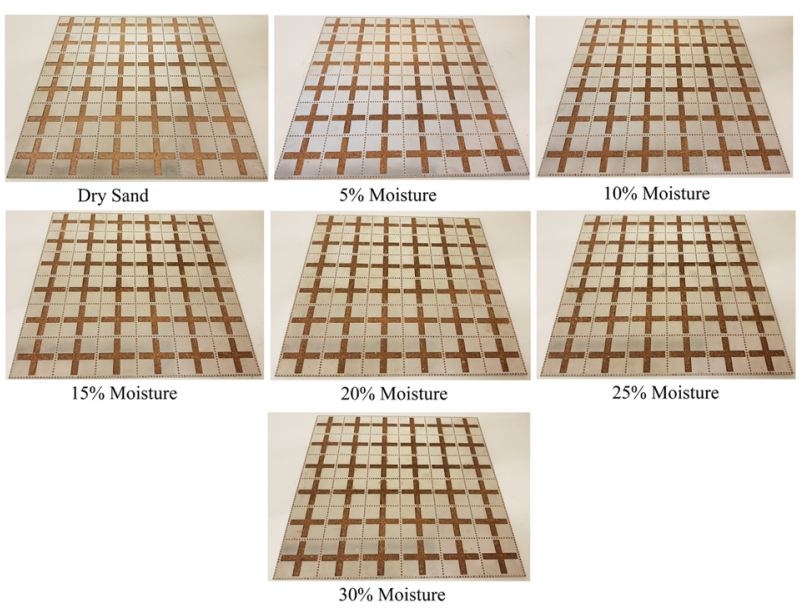

(a)

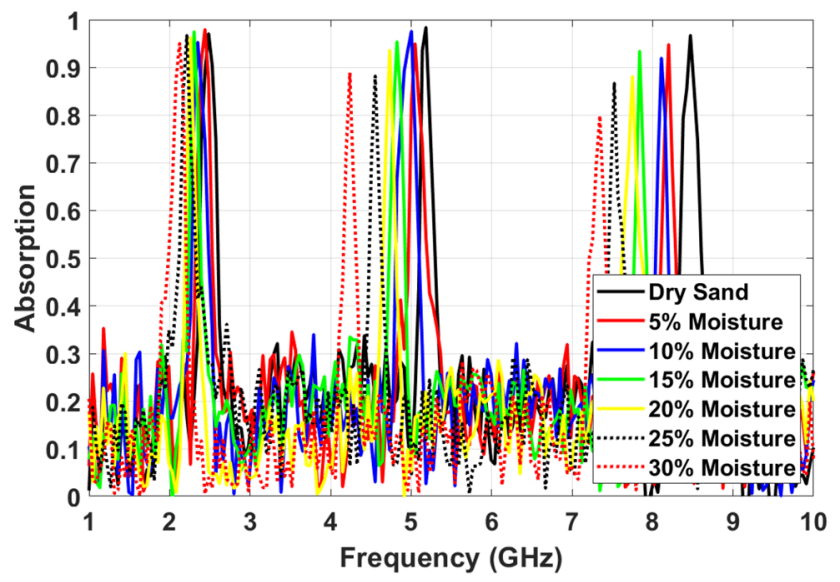

(b)

Figure 16. Measuring of sensing capability (a) Filling MPA channels by sand with different levels of moisture, (b) Changing absorption characteristics by varying the soil moisture.

$$
M C \%=\frac{W_{2}-W_{3}}{W_{3}-W_{1}} \times 100
$$

where $W_{1}, W_{2}$, and $W_{3}$ are the weight of the tin container, weight of moist soil plus the tin container, and weight of dried soil plus tin container, respectively. Figure 16a shows the structure with filled channels by soil at different levels of moisture. 


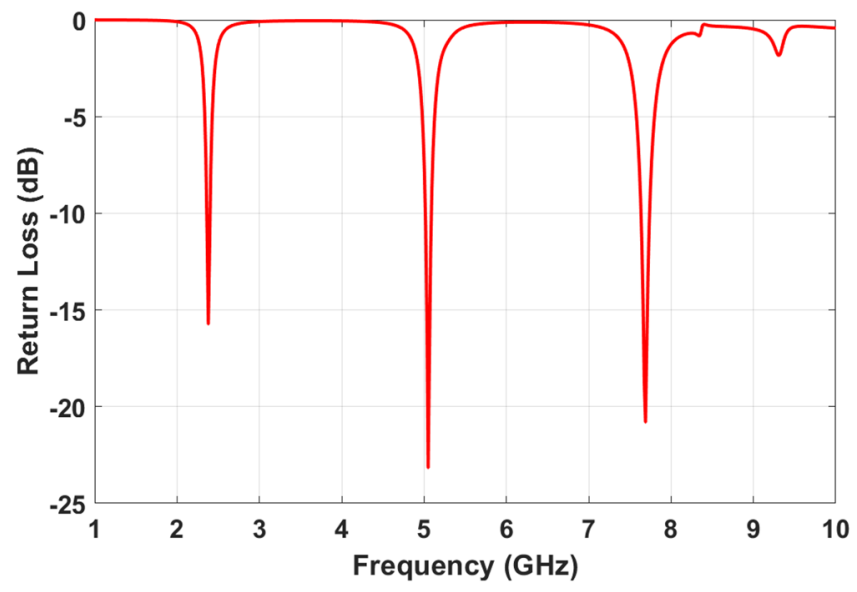

Figure 17. SIW-cavity MPA's return loss before etching channels.

The changes in the absorption peak frequency are shown in Fig. 16b that strongly follow the simulation results. $360 \mathrm{MHz}, 1 \mathrm{GHz}$, and $1.3 \mathrm{GHz}$ downshift at first, second, and third absorption frequencies have been observed for changing moisture content in sand, respectively. Based on $S_{\text {ave }}$ equation, the sensitivities of fabricated structure are $60.79,75.09$, and 59.21 for changing the water content in the sand from 0 to $30 \%$. Moreover, increasing the soil moisture level leads to the changing absorption ratio. As can be seen in Fig. 16b, the absorption ratio decreases from 98 to $91 \%$ at the first absorption frequency, from 98 to $85 \%$ at the second absorption frequency, and from 98 to $75 \%$ at the third absorption frequency when the sand moisture changes from 0 to $30 \%$.

\section{Discussion}

In this paper, a SIW cavity-based MPA has been introduced to implement a highly sensitive soil moisture sensor with an expected long operational lifetime. There is no need for an external power supply in the proposed sensor to sense soil moisture wirelessly. Furthermore, it can monitor the absorption characteristics from a distance by transmitting RF signals. Therefore, the proposed sensor is a promising candidate for remote sensing. Considering the effective medium theory and cavity principles, two perpendicular slots have been etched in the substrate to add the soil into the sensor structure. Furthermore, the SIW structure has been used to reduce the cost and complexity of the fabrication process. The sensing capability of the proposed sensor has been investigated for sand and loamy soil. The proposed sensor's resolution is $10 \mathrm{MHz}, 20 \mathrm{MHz}$, and $60 \mathrm{MHz}$ per percent for increasing the sand moisture. For loamy, the resolution becomes $7.5 \mathrm{MHz}, 22.5 \mathrm{MHz}$, and $52.5 \mathrm{MHz}$ per percent at three resonance frequency, respectively. Besides the absorption frequency shift, the absorption ratio can be considered a sensing parameter due to the down-scaling by increasing the soil moisture level. Based on the simulation results, $17 \%$ and $52 \%$ drops have been observed for changing sand moisture from 0 to $30 \%$ at second and third resonance frequencies. On the other hand, $23 \%$ and $54 \%$ reductions have been shown for changing loamy soil moisture from 0 to $40 \%$. Ultimately, an array of $6 \times 6$ has been fabricated on the FR- 4 substrate. Measurements follow the simulation result that confirms the correctness of the design procedure.

\section{Methods}

All simulations to design the proposed metamaterial absorber have been performed in commercial software CST STUDIO SUITE 2019 using a frequency-domain solver. The periodic boundary is applied for the unit cell to satisfy the metamaterial structure requirement. Parametric studies have been done under the normal incident angle to find optimum values for the proposed MPA parameters. Floqute port has been applied in simulation to facilitate changing incident and polarization angles to investigate structure sensitivity against different incident waves. The absorption characteristics have been extracted from Eq. (1) using return loss and transmission coefficient. Figure 17 shows the amount of reflected waves from MPA's surface under normal incident waves before etching slots.

\section{Measurement}

S-parameters have been used to calculate the absorption characteristics of the proposed MPA. These parameters were measured by implementing the described setup in Fig. 14b. A four ports ROHDE \& SCHWARZ ZVA40 vector network analyzer (VNA) and two ROHDE \& SCHWARZ HF907 dual-ridged horn reference antennas were used to perform measurements. One of the antennas has been connected to port one as a signal transmitter with a specified power of $-10 \mathrm{dBm}$. The other horn antenna performs to receive reflected signals from the MPA surface. Furthermore, the final setup was surrounded by wedge-trapped absorbing material to minimize the wave reflection in all directions. Before measuring S-parameter, the setup has been calibrated using a special calibration kit to neglect the effects of coaxial lines and connectors. The base level of transmission coefficient between antennas in the proposed setup must be specified by performing similar measurement scenarios using a metal plate with the same MPA size. These results were considered base level to compare with S-parameter and reduce the non-ideal testing setup's noise. In order to measure absorptivity at different incident angles, two 
antennas were moved equally around a circle with a Radius of $1 \mathrm{~m}$ from the center of MPA. The $\theta$ is the angle between the perpendicular axis to the center of MPA and horn antenna in the azimuth plane. In the sequence of measuring absorptivity at different polarization angles, either horn antennas or MPA structure need to be rotated perpendicular around the perpendicular axis.

Received: 7 December 2020; Accepted: 16 February 2021

Published online: 30 March 2021

\section{References}

1. Landy, N. I., Sajuyigbe, S., Mock, J. J., Smith, D. R. \& Padilla, W. J. Perfect metamaterial absorber. Physical review letters 100, 207402 (2008).

2. Wang, B.-X. Quad-band terahertz metamaterial absorber based on the combining of the dipole and quadrupole resonances of two srrs. IEEE J. Sel. Top. Quantum Electron 23, 1-7 (2016).

3. Gunduz, O. \& Sabah, C. Polarization angle independent perfect multiband metamaterial absorber and energy harvesting application. J. Comput. Electron 15, 228-238 (2016).

4. Tofigh, F., Amiri, M., Shariati, N., Lipman, J. \& Abolhasan, M. Polarization-insensitive metamaterial absorber for crowd estimation based on electromagnetic energy measurements. IEEE Trans. Antennas Propag. 68, 1458-1467 (2019).

5. Wang, B.-X., Wang, G.-Z., Sang, T. \& Wang, L.-L. Six-band terahertz metamaterial absorber based on the combination of multipleorder responses of metallic patches in a dual-layer stacked resonance structure. Sci. Rep. 7, 41373 (2017).

6. Long, C. et al. Broadening the absorption bandwidth of metamaterial absorbers by transverse magnetic harmonics of 210 mode. Sci. Rep. 6, 21431 (2016).

7. Amiri, M., Tofigh, F., Shariati, N., Lipman, J. \& Abolhasan, M. Wide-angle metamaterial absorber with highly insensitive absorption for te and tm modes. Sci. Rep. 10, 1-13 (2020).

8. Amiri, M., Tofigh, F., Shariati, N., Lipman, J. \& Abolhasan, M. Miniature tri-wideband sierpinski-minkowski fractals metamaterial perfect absorber. IET Microwav. Antennas Propag. 13, 991-996 (2019).

9. Hoa, N. T. Q., Tuan, T. S., Hieu, L. T. \& Giang, B. L. Facile design of an ultra-thin broadband metamaterial absorber for c-band applications. Sci. Rep. 9, 1-9 (2019).

10. Tofigh, F., Amiri, M., Shariati, N., Lipman, J. \& Abolhasan, M. Low-frequency metamaterial absorber using space-filling curve. J. Electron. Mater 48, 6451-6459 (2019).

11. Wang, H., Yang, Y. \& Wang, L. Switchable wavelength-selective and diffuse metamaterial absorber/emitter with a phase transition spacer layer. Appl. Phys. Lett 105, 071907 (2014).

12. Diem, M., Koschny, T. \& Soukoulis, C. M. Wide-angle perfect absorber/thermal emitter in the terahertz regime. Phys. Rev. B 79, 033101 (2009).

13. Yuan, S., Yang, R., Xu, J., Wang, J. \& Tian, J. Photoexcited switchable single-/dual-band terahertz metamaterial absorber. Mater. Res. Express 6, 075807 (2019).

14. Huang, T.-Y. et al. Experimental realization of ultrathin, double-sided metamaterial perfect absorber at terahertz gap through stochastic design process. Sci. Rep. 5, 18605 (2015).

15. Zhang, Y. et al. Independently tunable dual-band perfect absorber based on graphene at mid-infrared frequencies. Sci. Rep. 5, 18463 (2015).

16. Hajizadegan, M., Ahmadi, V. \& Sakhdari, M. Design and analysis of ultrafast and tunable all optical metamaterial switch enhanced by metal nanocomposite. J. Light. Technol. 31, 1877-1883 (2013).

17. Gong, Y. et al. Highly flexible all-optical metamaterial absorption switching assisted by kerr-nonlinear effect. Optics express 19, 10193-10198 (2011).

18. Almoneef, T. S., Erkmen, F. \& Ramahi, O. M. Harvesting the energy of multi-polarized electromagnetic waves. Sci. Rep. 7, 1-14 (2017).

19. Ghaderi, B., Nayyeri, V., Soleimani, M. \& Ramahi, O. M. Pixelated metasurface for dual-band and multi-polarization electromagnetic energy harvesting. Sci. Rep. 8, 1-12 (2018).

20. Niknam, S., Yazdi, M. \& Amlashi, S. B. enhanced ultra-sensitive metamaterial resonance sensor based on double corrugated metal stripe for terahertz sensing. Sci. Rep. 9, 1-9 (2019).

21. Zou, H. \& Cheng, Y. Design of a six-band terahertz metamaterial absorber for temperature sensing application. Optical Materials 88, 674-679 (2019).

22. Hasan, D. \& Lee, C. Hybrid metamaterial absorber platform for sensing of co2 gas at mid-ir. Advanced Science 5, 1700581 (2018).

23. Saadeldin, A. S., Hameed, M. F. O., Elkaramany, E. M. \& Obayya, S. S. Highly sensitive terahertz metamaterial sensor. IEEE Sensors J.IEEE Sensors J. 19, 7993-7999 (2019).

24. Ren, Z., Lin, Z., Zhi, X. \& Li, M. Double-layer broadband perfect metamaterial absorber and its potential for refractive index sensing. Opt. Mater. 99, 109575 (2020).

25. Levy, O. \& Stroud, D. Maxwell garnett theory for mixtures of anisotropic inclusions: Application to conducting polymers. Physical Review B 56, 8035 (1997).

26. Chang, C.-Y. et al. Flexible localized surface plasmon resonance sensor with metal-insulator-metal nanodisks on pdms substrate. Sci. Rep. 8, 1-8 (2018).

27. Kim, H. K., Lee, D. \& Lim, S. A fluidically tunable metasurface absorber for flexible large-scale wireless ethanol sensor applications. Sensors 16, 1246 (2016).

28. Zhang, Y., Zhao, J., Cao, J. \& Mao, B. Microwave metamaterial absorber for non-destructive sensing applications of grain. Sensors 18, 1912 (2018).

29. Sabah, C. et al. Perfect metamaterial absorber with polarization and incident angle independencies based on ring and cross-wire resonators for shielding and a sensor application. Opt. Commun. 322, 137-142 (2014).

30. Sabah, C., Turkmen-Kucuksari, O. \& Turhan-Sayan, G. Metamaterial absorber-based sensor embedded into x-band waveguide. Electron. Lett. 50, 1074-1076 (2014).

31. Bakır, M., Karaaslan, M., Dinçer, F., Delihacioglu, K. \& Sabah, C. Tunable perfect metamaterial absorber and sensor applications. J. Mater. Sci. Mater. Electron. 27, 12091-12099 (2016).

32. Bakir, M. et al. Sensory applications of resonator based metamaterial absorber. Optik 168, 741-746 (2018).

33. Bakır, M., Karaaslan, M., Unal, E., Akgol, O. \& Sabah, C. Microwave metamaterial absorber for sensing applications. Opto Electron. Rev. 25, 318-325 (2017).

34. Guo, Q.-Y. \& Wong, H. Wideband and high-gain fabry-pérot cavity antenna with switched beams for millimeter-wave applications. IEEE Trans. Antennas Propag. 67, 4339-4347 (2019).

35. Lima, E. B., Costa, J. R. \& Fernandes, C. A. Multiple-beam focal-plane dual-band fabry-pérot cavity antenna with reduced beam degradation. IEEE Trans. Antennas Propag. 67, 4348-4356 (2019).

36. Liu, X., Katehi, L. P., Chappell, W. J. \& Peroulis, D. High-q tunable microwave cavity resonators and filters using soi-based rf mems tuners. J. Microelectromech. Syst. 19, 774-784 (2010). 
37. Abdelfattah, M. \& Peroulis, D. High- $q$ tunable evanescent-mode cavity siw resonators and filters with contactless tuners. IEEE Trans. Microw. Theory Tech. 67, 3661-3672 (2019).

38. Bastioli, S., Snyder, R. V. \& Tomassoni, C. Over-moded transverse magnetic cavity filters for narrowband millimeter-wave applications. IEEE Microw. Wirel. Components Lett. 29, 321-323 (2019).

39. Wei, Z. et al. A high-sensitivity microfluidic sensor based on a substrate integrated waveguide re-entrant cavity for complex permittivity measurement of liquids. Sensors 18, 4005 (2018).

40. Seo, Y., Memon, M. U. \& Lim, S. Microfluidic eighth-mode substrate-integrated-waveguide antenna for compact ethanol chemical sensor application. IEEE Trans. Antennas Propag. 64, 3218-3222 (2016).

41. Salim, A., Kim, S.-H., Park, J. Y. \& Lim, S. Microfluidic biosensor based on microwave substrate-integrated waveguide cavity resonator. J. Sensors 2018 (2018).

42. Memon, M. U. \& Lim, S. Microfluidic high-q circular substrate-integrated waveguide (siw) cavity for radio frequency (rf) chemical liquid sensing. Sensors 18, 143 (2018).

43. Power, J. The effect of moisture on fertilizer nitrogen immoblization in grasslands. Soil Sci. Soc. Am. J. 31, 223-226 (1967).

44. Koschny, T. et al. Impact of inherent periodic structure on effective medium description of left-handed and related metamaterials. Phys. Rev. B 71, 245105 (2005).

45. Pozar, D. Microwave Engineering 4th edn. (Wiley, New York, 2011).

46. Deslandes, D. \& Wu, K. Design consideration and performance analysis of substrate integrated waveguide components. In 2002 32nd European Microwave Conference, 1-4 (IEEE, 2002).

47. Xu, F. \& Wu, K. Guided-wave and leakage characteristics of substrate integrated waveguide. IEEE Trans. Microwav. Theory Tech. 53, 66-73 (2005).

48. Smith, D., Vier, D., Koschny, T. \& Soukoulis, C. Electromagnetic parameter retrieval from inhomogeneous metamaterials. Phys. Rev. E 71, 036617 (2005).

49. Mahmud, S., Islam, S. S., Almutairi, A. F. \& Islam, M. T. A wide incident angle, ultrathin, polarization-insensitive metamaterial absorber for optical wavelength applications. IEEE Access 8, 129525-129541 (2020).

50. Mahmud, S. et al. Design and parametric analysis of a wide-angle polarization-insensitive metamaterial absorber with a star shape resonator for optical wavelength applications. Results in Physics 18, 103259 (2020).

51. Cihlar, J. \& Ulaby, F. T. Dielectric properties of soils as a function of moisture content. In CRES Tech. Rep. (1974).

52. Bobrov, P. P., Repin, A. V. \& Rodionova, O. V. Wideband frequency domain method of soil dielectric property measurements. IEEE Trans. Geosci. Remote. Sens. 53, 2366-2372 (2014).

53. Withayachumnankul, W., Jaruwongrungsee, K., Tuantranont, A., Fumeaux, C. \& Abbott, D. Metamaterial-based microfluidic sensor for dielectric characterization. Sensors Actuators A Phys. 189, 233-237 (2013).

54. Ebrahimi, A., Withayachumnankul, W., Al-Sarawi, S. \& Abbott, D. High-sensitivity metamaterial-inspired sensor for microfluidic dielectric characterization. IEEE Sensors J. 14, 1345-1351 (2013).

55. Kim, H. K., Yoo, M. \& Lim, S. Novel ethanol chemical sensor using microfluidic metamaterial. In 2015 IEEE International Symposium on Antennas and Propagation \& USNC/URSI National Radio Science Meeting, 1358-1359 (IEEE, 2015).

56. Vélez, P. et al. Microwave microfluidic sensor based on a microstrip splitter/combiner configuration and split ring resonators (SRRS) for dielectric characterization of liquids. IEEE Sensors J. 17, 6589-6598 (2017).

57. Ling, K. et al. Microfluidic tunable inkjet-printed metamaterial absorber on paper. Opt. Express 23, 110-120 (2015).

58. Salim, A. \& Lim, S. Review of recent metamaterial microfluidic sensors. Sensors 18, 232 (2018).

59. Department of Sustainable Natural Resources. Soil survey standard test method soil moisture content. https://www.environment. nsw.gov.au/resources/soils/testmethods/mc.pdf.

\section{Acknowledgements}

We would like to acknowledge the partial support of Food Agility CRC Ltd, funded under Commonwealth Government CRC Program. The CRC Program supports industry-led collaboration between industry, research and the community.

\section{Author contributions}

M.A. designed the proposed structure, and carried out the full-wave simulations, and analysed the results, and conducted the experiments, and wrote the manuscript. M.A., N.S. and J.L. reviewed and contributed to revising the manuscript.

\section{Competing interests}

The authors declare no competing interests.

\section{Additional information}

Correspondence and requests for materials should be addressed to M.A.

Reprints and permissions information is available at www.nature.com/reprints.

Publisher's note Springer Nature remains neutral with regard to jurisdictional claims in published maps and institutional affiliations.

(c) (i) Open Access This article is licensed under a Creative Commons Attribution 4.0 International cc) License, which permits use, sharing, adaptation, distribution and reproduction in any medium or format, as long as you give appropriate credit to the original author(s) and the source, provide a link to the Creative Commons licence, and indicate if changes were made. The images or other third party material in this article are included in the article's Creative Commons licence, unless indicated otherwise in a credit line to the material. If material is not included in the article's Creative Commons licence and your intended use is not permitted by statutory regulation or exceeds the permitted use, you will need to obtain permission directly from the copyright holder. To view a copy of this licence, visit http://creativecommons.org/licenses/by/4.0/.

(C) The Author(s) 2021 